\title{
New existence results on positive solutions of four-point integral type BVPs for coupled multi-term fractional differential equations
}

\author{
Xiaohui Yang ${ }^{1}$ Yuji Liu $^{2}$
}

Received: 29 July 2016/ Accepted: 26 October 2016/Published online: 10 November 2016

(c) The Author(s) 2016. This article is published with open access at Springerlink.com

\begin{abstract}
In this article, we establish some new existence results on positive solutions of a four-point integral boundary value problem for coupled nonlinear multi-term fractional differential equations. Our analysis rely on the well known fixed point theorems. Numerical examples are given to illustrate the main theorems.
\end{abstract}

Keywords Four-point integral boundary value problem . Multi-term fractional differential system · NonCarathéodory function · Fixed-point theorem

\section{Mathematics Subject Classification 92D25 - 34A37 . $34 \mathrm{~K} 15$}

\section{Introduction}

Fractional differential systems have many applications in modeling of physical and chemical processes and in engineering $[3,14,19]$, and have been of great interest recently. In

Supported by the Natural Science Foundation of Guangdong province (No. S2011010001900) and Natural science research project for colleges and universities of Guangdong Province (No: 2014KTSCX126).

Yuji Liu

liuyuji888@sohu.com

Xiaohui Yang

xiaohuiyang@sohu.com

1 Department of Computer, Guangdong Police College, Guangzhou 510230, People's Republic of China

2 Department of Mathematics, Guangdong University of Finance and Economics, Guangzhou 510320, People's Republic of China its turn, mathematical aspects of studies on fractional differential systems were discussed by many authors, see the text books [5, 15] and papers [1, 6, 8, 13, 16, 17, 20, 21, 23-26]. A survey concerning the studies on solvability of two-point or four-point boundary value problems for fractional differential systems was given in [11].

In this paper, we discuss the existence of positive solutions of the following four-point integral type boundary value problem for the multi-term fractional differential system

$$
\left\{\begin{array}{l}
D_{0^{+}}^{\alpha} u(t)+p(t) f\left(t, v(t), D_{0^{+}}^{n} v(t)\right)=0, a . e ., t \in(0,1), \\
D_{0^{+}}^{\beta} v(t)+q(t) g\left(t, u(t), D_{0^{+}}^{m} u(t)\right)=0, a . e ., t \in(0,1), \\
\lim _{t \rightarrow 0} t^{2-\alpha} u(t)-a u(\xi)=\int_{0}^{1} \phi_{1}\left(t, v(t), D_{0^{+}}^{n} v(t)\right) \mathrm{d} t \\
u(1)-b u(\eta)=\int_{0}^{1} \psi_{1}\left(t, v(t), D_{0^{+}}^{n} v(t)\right) \mathrm{d} t \\
\lim _{t \rightarrow 0} t^{2-\beta} v(t)-c v(\xi)=\int_{0}^{1} \phi_{2}\left(t, u(t), D_{0^{+}}^{m} u(t)\right) \mathrm{d} t \\
v(1)-d v(\eta)=\int_{0}^{1} \psi_{2}\left(t, u(t), D_{0^{+}}^{m} u(t)\right) \mathrm{d} t
\end{array}\right.
$$

where

(i) $1<\alpha, \beta \leq 2, \alpha-1<m<\alpha$ and $\beta-1<n<\beta, D_{0^{+}}^{*}$ is the standard Riemann-Liouville differential derivative of order $*>0$ with the starting point 0 ,

(ii) $0<\xi \leq \eta<1$ and $a, b, c, d \geq 0$,

(iii) $\quad p, q:(0,1) \rightarrow \mathbb{R}, p$ satisfies that there exist numbers $k_{1}, l_{1}$ such that $k_{1}>-1, \alpha-m+l_{1}>0$, $2+k_{1}+l_{1}>0$ and $|p(t)|<t^{k_{1}}(1-t)^{l_{1}}$ for $t \in(0,1), q$ satisfies that there exist numbers $k_{2}, l_{2}$ such that $k_{2}>-1, \beta-n+l_{2}>0,2+$ $k_{2}+l_{2}>0$ and $|q(t)|<t^{k_{2}}(1-t)^{l_{2}}$ for $t \in(0,1)$, with $p(t) \not \equiv 0$ and $q(t) \not \equiv 0$ on $(0,1)$,

(iv) $f, g, \phi_{i}, \psi_{i}:(0,1) \times[0,+\infty) \times \mathbb{R} \rightarrow[0,+\infty), f$ is a strong $(n, \beta)$-Carathéory function and $g$ is a strong 
$(m, \alpha)$-Carathéory function with $f(t, 0,0) \not \equiv 0$ and $g(t, 0,0) \not \equiv 0$ on $(0,1), \phi_{1}, \psi_{1}$ are $(n, \beta)$-Carathéory functions and $\phi_{2}, \psi_{2}$ are $(m, \alpha)$-Carathéory functions.

A pair of functions $(x, y)$ is called a solution of BVP (1) if $x, y \in C^{0}(0,1]$ and $x, y$ satisfy all equations in (1). We obtain the results on solutions of BVP(1) using Schauder's fixed point theorem in Banach spaces. The salient features of this study are as follows:

(a) the fractional differential equations in (1) are multiterm ones and their nonlinearities depend on the lower order fractional derivatives with order greater than $\alpha-1$ and $\beta-1$;

(b) instead of the condition $u(0)=0, v(0)=0$ we consider integral boundary conditions which are more suitable as $D_{0^{+}}^{\alpha} x(t)=0$ with $\alpha \in(1,2)$ implies $x(t)=c t^{\alpha-1}$ and obviously $x$ is not continuous at $t=0$ while $\lim _{t \rightarrow 0} t^{2-\alpha} x(t)$ exists;

(c) $\mathrm{BVP}(1)$ is a generalized form of known ones in references $[4,7,9,10,21]$, the positive solutions of BVP(1) obtained are unbounded (discontinuous at $t=0$ ) which are different from those ones (continuous on [0,1]) in [1, 8, 23, 24];

(d) this paper is a complement of [11] in which the existence of positive solutions of BVP(1) was studied under the assumptions $m \in(0, \alpha-1]$ and $n \in(0, \beta-1]$ while $m \in(\alpha-1, \alpha), n \in(\beta-1, \beta)$ are supposed in this paper.

The remainder of this paper is arranged as follows: in Sect. 2, we present preliminary results; in Sect. 3, the main results are presented; and two examples are given in Sect. 4 to illustrate the main results.

\section{Preliminary results}

For the convenience of readers, we present here the necessary definitions from fixed point theory and fractional calculus theory.

Definition 2.1 [2] Let $X$ be a Banach space. An operator $T: X \rightarrow X$ is completely continuous if it is continuous and maps bounded sets into pre-compact sets (or relatively compact sets).

Definition 2.2 [15] The left Riemann-Liouville fractional integral (left forward) of order $\alpha>0$ of a function $f:(0, \infty) \rightarrow \mathbb{R}$ is given by

$I_{0+}^{\alpha} f(t)=\frac{1}{\Gamma(\alpha)} \int_{0}^{t}(t-s)^{\alpha-1} f(s) \mathrm{d} s, \quad t>0$

provided that the right-hand side exists.
Definition 2.3 [15] The left Riemann-Liouville fractional derivative (left farward) of order $\alpha>0$ of a continuous function $f:(0, \infty) \rightarrow \mathbb{R}$ is given by

$D_{0^{+}}^{\alpha} f(t)=\frac{1}{\Gamma(n-\alpha)} \frac{\mathrm{d}^{n}}{\mathrm{~d} t^{n}} \int_{0}^{t} \frac{f(s)}{(t-s)^{\alpha-n+1}} \mathrm{~d} s, \quad t>0$

where $n-1<\alpha<n$, provided that the right-hand side exists.

Definition $2.4 h:(0,1) \times \mathbb{R} \times \mathbb{R} \rightarrow \mathbb{R}$ is called a $(m, \alpha)$-Carathédory function if it satisfies

(i) $\quad t \rightarrow h\left(t, t^{\alpha-2} x, t^{2+m-\alpha} y\right)$ is measurable on $(0,1)$ for all $(x, y) \in \mathbb{R}^{2}$,

(ii) $(x, y) \rightarrow h\left(t, t^{\alpha-2} x, t^{2+m-\alpha} y\right)$ is continuous for a.e. $t \in(0,1)$,

(iii) for each $r>0$, there exists nonnegative number $M_{r}$ such that $|u|,|v| \leq r$ imply

$$
\left|h\left(t, t^{\alpha-2} x, t^{2+m-\alpha} y\right)\right| \leq M_{r}, \quad \text { a.e.t } \in(0,1) .
$$

Definition 2.5 $h:(0,1) \times \mathbb{R} \times \mathbb{R} \rightarrow \mathbb{R}$ is called a $(m, \alpha)$-Carathédory function if it satisfies

(i) $t \rightarrow h\left(t, t^{\alpha-2} x, t^{2+m-\alpha} y\right)$ is measurable on $(0,1)$ for all $(x, y) \in \mathbb{R}^{2}$,

(ii) $(x, y) \rightarrow h\left(t, t^{\alpha-2} x, t^{2+m-\alpha} y\right)$ is continuous for a.e. $t \in(0,1)$,

(iii) for each $r>0$, there exists nonnegative function $\phi_{r} \in L^{1}(0,1)$ such that $|u|,|v| \leq r$ imply

$$
\left|h\left(t, t^{\alpha-2} x, t^{2+m-\alpha} y\right)\right| \leq \phi_{r}(t), \quad \text { a.e.t } \in(0,1) \text {. }
$$

Lemma 2.1 [15] Let $n-1 \leq \alpha<n, \quad u \in C^{0}(0, \infty) \bigcap$ $L^{1}(0, \infty)$. Then

$$
I_{0+}^{\alpha} D_{0+}^{\alpha} u(t)=u(t)+C_{1} t^{\alpha-1}+C_{2} t^{\alpha-2}+\cdots+C_{n} t^{\alpha-n},
$$

where $C_{i} \in R, i=1,2, \ldots n$

\section{Choose}

$X=\left\{x:(0,1] \rightarrow \mathbb{R}^{x, D_{0^{+}}^{m} x \in C^{0}(0,1] \text { the following limits exist }} \begin{array}{c}\lim _{t \rightarrow 0} t^{2-\alpha} x(t), \lim _{t \rightarrow 0} t^{2+m-\alpha} D_{0^{+}}^{m} x(t)\end{array}\right\}$

with the norm

$\|x\|=\|x\|_{X}=\max \left\{\sup _{t \in(0,1]} t^{2-\alpha}|x(t)|, \sup _{t \in(0,1]} t^{2+m-\alpha}\left|D_{0^{+}}^{m} x(t)\right|\right\}$

for $x \in X$. It is easy to show that $X$ is a real Banach space. Choose

$Y=\left\{y:(0,1] \rightarrow \mathbb{R}^{y, D_{0^{+}}^{n} y \in C^{0}(0,1] \text { the following limits exist }} \begin{array}{c}\lim _{t \rightarrow 0} t^{2-\beta} y(t), \lim _{t \rightarrow 0} t^{2+n-\beta} D_{0^{+}}^{n} y(t)\end{array}\right\}$ 
with the norm

$\|y\|=\|y\|_{Y}=\max \left\{\sup _{t \in(0,1]} t^{2-\beta}|y(t)|, \sup _{t \in(0,1]} t^{2+n-\beta}\left|D_{0^{+}}^{n} y(t)\right|\right\}$

for $y \in Y$. It is easy to show that $Y$ is a real Banach space.

Thus, $(X \times Y,\|\cdot\|)$ is Banach space with the norm defined by

$\|(x, y)\|=\max \left\{\|x\|=\|x\|_{X},\|y\|=\|y\|_{Y}\right\}$ for

$(x, y) \in X \times Y$.

For a function $x:(0,1] \rightarrow \mathbb{R}$, a number $m$ and a function $F:(0,1) \times \mathbb{R}^{2} \rightarrow \mathbb{R}$, denote $F_{m, x}(t)=F\left(t, x(t), D_{0^{+}}^{m} x(t)\right)$.

Denote

$\mu_{1}=a \xi^{\alpha-1}, \quad v_{1}=1-a \xi^{\alpha-2}, \quad \omega_{1}=1-b \eta^{\alpha-1}$,

$\lambda_{1}=1-b \eta^{\alpha-2}, \quad \Delta=\mu_{1} \lambda_{1}+v_{1} \omega_{1}$

$\mu_{2}=c \xi^{\beta-1}, \quad v_{2}=1-c \xi^{\beta-2}, \quad \omega_{2}=1-d \eta^{\beta-1}$,

$\lambda_{2}=1-d \eta^{\beta-2}, \quad \nabla=\mu_{2} \lambda_{2}+v_{2} \omega_{2}$.

Lemma 2.2 (Lemma 2.6 in [11]) Suppose that $\Delta \neq 0$ and

(B0) $h \in C^{0}(0,1)$ and there exist $k>-1$ and $l \leq 0$ such that $2+l+k>0$ and $|h(t)| \leq t^{k}(1-t)^{l}$ for all $t \in(0,1)$.

Then $x \in X$ is a solution of problem

$$
\left\{\begin{array}{l}
D^{\alpha} x(t)+h(t)=0,0<t<1 \\
\lim _{t \rightarrow 0} t^{2-\alpha} x(t)-a x(\xi)=M, x(1)-b x(\eta)=N
\end{array}\right.
$$

if and only if $x \in X$ satisfies

$$
\begin{aligned}
x(t)= & \frac{v_{1} t^{\alpha-1}+\mu_{1} t^{\alpha-2}}{\Delta} N+\frac{\omega_{1} t^{\alpha-2}-\lambda_{1} t^{\alpha-1}}{\Delta} M \\
& -\int_{0}^{t} \frac{(t-s)^{\alpha-1}}{\Gamma(\alpha)} h(s) \mathrm{d} s+\frac{v_{1} t^{\alpha-1}+\mu_{1} t^{\alpha-2}}{\Delta} \\
& \times \int_{0}^{1} \frac{(1-s)^{\alpha-1}}{\Gamma(\alpha)} h(s) \mathrm{d} s \\
& -\frac{b v_{1} t^{\alpha-1}+b \mu_{1} t^{\alpha-2}}{\Delta} \int_{0}^{\eta} \frac{(\eta-s)^{\alpha-1}}{\Gamma(\alpha)} h(s) \mathrm{d} s \\
& +\frac{a \lambda_{1} t^{\alpha-1}-a \omega_{1} t^{\alpha-2}}{\Delta} \int_{0}^{\xi} \frac{(\xi-s)^{\alpha-1}}{\Gamma(\alpha)} h(s) \mathrm{d} s .
\end{aligned}
$$

Lemma 2.3 (Lemma 2.7 in [11]) Suppose that $\nabla \neq 0$ and (B0) holds. Then $y \in Y$ is a solution of problem

$$
\left\{\begin{array}{l}
D^{\beta} y(t)+h(t)=0,0<t<1, \\
\lim _{t \rightarrow 0} t^{2-\beta} y(t)-c y(\xi)=M, y(1)-d y(\eta)=N
\end{array}\right.
$$

if and only if $y \in Y$ satisfies

$$
\begin{aligned}
y(t)= & \frac{v_{2} t^{\beta-1}+\mu_{2} t^{\beta-2}}{\nabla} N+\frac{\omega_{2} t^{\beta-2}-\lambda_{2} t^{\beta-1}}{\nabla} M \\
& -\int_{0}^{t} \frac{(t-s)^{\beta-1}}{\Gamma(\beta)} h(s) \mathrm{d} s+\frac{v_{2} t^{\beta-1}+\mu_{2} t^{\beta-2}}{\nabla} \\
& \times \int_{0}^{1} \frac{(1-s)^{\beta-1}}{\Gamma(\beta)} h(s) \mathrm{d} s \\
& -\frac{d \lambda_{2} t^{\beta-1}+d \mu_{2} t^{\beta-2}}{\nabla} \int_{0}^{\eta} \frac{(\eta-s)^{\beta-1}}{\Gamma(\beta)} h(s) \mathrm{d} s \\
& +\frac{c \lambda_{2} t^{\beta-1}-c \omega_{2} t^{\beta-2}}{\nabla} \int_{0}^{\xi} \frac{(\xi-s)^{\beta-1}}{\Gamma(\beta)} h(s) \mathrm{d} s .
\end{aligned}
$$

Define the operator $T$ on $X \times Y$, for $(x, y) \in X \times Y$, by $T(x, y)(t)=\left(\left(T_{1} y\right)(t),\left(T_{2} x\right)(t)\right)$ with

$$
\begin{aligned}
\left(T_{1} y\right)(t)= & \frac{v_{1} t^{\alpha-1}+\mu_{1} t^{\alpha-2}}{\Delta} \int_{0}^{1} \psi_{1 n, y}(s) \mathrm{d} s+\frac{\omega_{1} t^{\alpha-2}-\lambda_{1} t^{\alpha-1}}{\Delta} \\
& \times \int_{0}^{1} \phi_{1 n, y}(s) \mathrm{d} s \\
& -\int_{0}^{t} \frac{(t-s)^{\alpha-1}}{\Gamma(\alpha)} p(s) f_{n, y}(s) \mathrm{d} s+\frac{v_{1} t^{\alpha-1}+\mu_{1} t^{\alpha-2}}{\Delta} \\
& \times \int_{0}^{1} \frac{(1-s)^{\alpha-1}}{\Gamma(\alpha)} p(s) f_{n, y}(s) \mathrm{d} s \\
& -\frac{b v_{1} t^{\alpha-1}+b \mu_{1} t^{\alpha-2}}{\Delta} \int_{0}^{\eta} \frac{(\eta-s)^{\alpha-1}}{\Gamma(\alpha)} p(s) f_{n, x}(s) \mathrm{d} s \\
& +\frac{a \lambda_{1} t^{\alpha-1}-a \omega_{1} t^{\alpha-2}}{\Delta} \int_{0}^{\xi} \frac{(\xi-s)^{\alpha-1}}{\Gamma(\alpha)} p(s) f_{n, y}(s) \mathrm{d} s
\end{aligned}
$$

and

$$
\begin{aligned}
\left(T_{2} x\right)(t)= & \frac{v_{2} t^{\beta-1}+\mu_{2} t^{\beta-2}}{\nabla} \int_{0}^{1} \psi_{2 m, x}(s) \mathrm{d} s+\frac{\omega_{2} t^{\beta-2}-\lambda_{2} t^{\beta-1}}{\nabla} \\
& \times \int_{0}^{1} \phi_{2 m, x}(s) \mathrm{d} s \\
& -\int_{0}^{t} \frac{(t-s)^{\beta-1}}{\Gamma(\beta)} q(s) g_{m, x}(s) \mathrm{d} s+\frac{v_{2} t^{\beta-1}+\mu_{2} t^{\beta-2}}{\nabla} \\
& \times \int_{0}^{1} \frac{(1-s)^{\beta-1}}{\Gamma(\beta)} q(s) g_{m, x}(s) \mathrm{d} s \\
& -\frac{d v_{2} t^{\beta-1}+d \mu_{2} t^{\beta-2}}{\nabla} \int_{0}^{\eta} \frac{(\eta-s)^{\beta-1}}{\Gamma(\beta)} q(s) g_{m, x}(s) \mathrm{d} s \\
& +\frac{c \lambda_{2} t^{\beta-1}-c \omega_{2} t^{\beta-2}}{\nabla} \int_{0}^{\xi} \frac{(\xi-s)^{\beta-1}}{\Gamma(\beta)} q(s) g_{m, x}(s) \mathrm{d} s .
\end{aligned}
$$

By Lemmas 2.2 and 2.3, we have that $(x, y) \in X \times Y$ is a solution of $\operatorname{BVP}(8)$ if and only if $(x, y) \in X \times Y$ is a fixed point of $T$.

Lemma 2.4 Suppose that (i)-(iv) defined in Sect. 1 hold, $\Delta \neq 0$ and $\nabla \neq 0$. Then $T: X \times Y \rightarrow X \times Y$ is completely continuous. 
Proof We will prove that both $T_{1}$ and $T_{2}$ are completely continuous. The proof of the completeness of $T_{1}$ is divided into four steps and similarly we can prove that $T_{2}$ is completely continuous.

Step 1 Suppose that $\alpha-1<m<\alpha$. We prove that both $T_{1}$ : $Y \rightarrow X$ is well defined.

For $y \in Y$, there exits $r>0$ such that

$\max \left\{\sup _{t \in(0,1]} t^{2-\beta}|y(t)|, \sup _{t \in(0,1]} t^{2+n-\beta}\left|D_{0^{+}}^{n} y(t)\right| \mid\right\}<r$.

Then (iii) and (iv) imply that there exists a number $M_{r}>0$ and $\phi_{0}, \psi_{0} \in L^{1}(0,1)$ such that

$$
\begin{gathered}
\mid f\left(t, y(t), D_{0^{+}}^{n} y(t)|=| f\left(t, t^{\beta-2} t^{2-\beta} y(t),\right.\right. \\
\left.t^{\beta-n-2} t^{2+n-\beta} D_{0^{+}}^{n} y(t)\right) \mid \leq M_{r}, \\
\mid \phi_{1}\left(t, y(t), D_{0^{+}}^{n} y(t)\left|\leq \phi_{0}(t),\right| \psi_{1}\left(t, y(t), D_{0^{+}}^{n} y(t) \mid \leq \psi_{0}(t)\right.\right.
\end{gathered}
$$

for all $t \in(0,1)$. Then

$$
\begin{aligned}
& \left|\int_{0}^{t} \frac{(t-s)^{\alpha-1}}{\Gamma(\alpha)} p(s) f_{n, y}(s) \mathrm{d} s\right| \\
& \leq \int_{0}^{t} \frac{(t-s)^{\alpha-1}}{\Gamma(\alpha)} s^{k_{1}}(1-s)^{l_{1}} M_{r} \mathrm{~d} s \\
& \leq M_{r} \int_{0}^{t} \frac{(t-s)^{\alpha+l_{1}-1}}{\Gamma(\alpha)} s^{k_{1}} \mathrm{~d} s \\
& =M_{r} t^{\alpha+k_{1}+l_{1}} \int_{0}^{1} \frac{(1-w)^{\alpha+l_{1}-1}}{\Gamma(\alpha)} w^{k_{1}} \mathrm{~d} w \\
& =\frac{\mathbf{B}\left(\alpha+l_{1}, k_{1}+1\right) M_{r}}{\Gamma(\alpha)} t^{\alpha+k_{1}+l_{1}}, \\
& \int_{0}^{t} \frac{(t-s)^{\alpha-m-1}}{\Gamma(\alpha-m)} p(s) f_{n, y}(s) \mathrm{d} s \mid \\
& \quad \leq \int_{0}^{t} \frac{(t-s)^{\alpha-m-1}}{\Gamma(\alpha-m)} s^{k_{1}}(1-s)^{l_{1}} M_{r} \mathrm{~d} s \\
& \quad \leq M_{r} \int_{0}^{t} \frac{(t-s)^{\alpha-m+l_{1}-1}}{\Gamma(\alpha-m)} s^{k_{1}} \mathrm{~d} s \\
& \quad=M_{r} t^{\alpha-m+k_{1}+l_{1}} \int_{0}^{1} \frac{(1-w)^{\alpha-m+l_{1}-1}}{\Gamma(\alpha)} w^{k_{1}} \mathrm{~d} w \\
& \quad=\frac{\mathbf{B}\left(\alpha-m+l_{1}, k_{1}+1\right) M_{r}}{\Gamma(\alpha-m)} t^{\alpha-m+k_{1}+l_{1}} .
\end{aligned}
$$

On the other hand, note $D_{0^{+}}^{m} t^{\mu}=\frac{\Gamma(\mu+1)}{\Gamma(\mu+1-m)} t^{\mu-m}, \Gamma(0)=\infty$ with $\frac{1}{\Gamma(0)}=0$, we have

$$
\begin{aligned}
t^{2-\alpha}\left(T_{1} y\right)(t)=\frac{v_{1} t}{\Delta}+\mu_{1} & \int_{0}^{1} \psi_{1 n, y}(s) \mathrm{d} s+\frac{\omega_{1}-\lambda_{1} t}{\Delta} \int_{0}^{1} \phi_{1 n, y}(s) \mathrm{d} s \\
& -t^{2-\alpha} \int_{0}^{t} \frac{(t-s)^{\alpha-1}}{\Gamma(\alpha)} p(s) f_{n, y}(s) \mathrm{d} s+\frac{v_{1} t+\mu_{1}}{\Delta} \\
& \times \int_{0}^{1} \frac{(1-s)^{\alpha-1}}{\Gamma(\alpha)} p(s) f_{n, y}(s) \mathrm{d} s \\
& -\frac{b v_{1} t+b \mu_{1}}{\Delta} \int_{0}^{\eta} \frac{(\eta-s)^{\alpha-1}}{\Gamma(\alpha)} p(s) f_{n, x}(s) \mathrm{d} s \\
& +\frac{a \lambda_{1} t-a \omega_{1}}{\Delta} \int_{0}^{\xi} \frac{(\xi-s)^{\alpha-1}}{\Gamma(\alpha)} p(s) f_{n, y}(s) \mathrm{d} s,
\end{aligned}
$$

$$
\begin{aligned}
& t^{2+m-\alpha} D_{0^{+}}^{m}\left(T_{1} y\right)(t) \\
& =\frac{v_{1} \frac{\Gamma(\alpha)}{\Gamma(\alpha-m)} t+\mu_{1} \frac{\Gamma(\alpha-1)}{\Gamma(\alpha-m-1)}}{\Delta} \int_{0}^{1} \psi_{1 n, y}(s) \mathrm{d} s \\
& \quad+\frac{\omega_{1} \frac{\Gamma(\alpha-1)}{\Gamma(\alpha-m-1)}-\lambda_{1} \frac{\Gamma(\alpha)}{\Gamma(\alpha-m)} t}{\Delta} \int_{0}^{1} \phi_{1 n, y}(s) \mathrm{d} s-t^{2+m-\alpha} \\
& \quad \times \int_{0}^{t} \frac{(t-s)^{\alpha-m-1}}{\Gamma(\alpha-m)} p(s) f_{n, y}(s) \mathrm{d} s \\
& +\frac{v_{1} \frac{\Gamma(\alpha)}{\Gamma(\alpha-m)} t+\mu_{1} \frac{\Gamma(\alpha-1)}{\Gamma(\alpha-m-1)}}{\Delta} \int_{0}^{1} \frac{(1-s)^{\alpha-1}}{\Gamma(\alpha)} p(s) f_{n, y}(s) \mathrm{d} s \\
& \quad-\frac{b v_{1} \frac{\Gamma(\alpha)}{\Gamma(\alpha-m)} t+b \mu_{1} \frac{\Gamma(\alpha-1)}{\Gamma(\alpha-m-1)}}{\Delta} \int_{0}^{\eta} \frac{(\eta-s)^{\alpha-1}}{\Gamma(\alpha)} p(s) f_{n, x}(s) \mathrm{d} s \\
& +\frac{a \lambda_{1} \frac{\Gamma(\alpha)}{\Gamma(\alpha-m)} t-a \omega \omega_{1} \frac{\Gamma(\alpha-1)}{\Gamma(\alpha-m-1)}}{\Delta} \int_{0}^{\xi} \frac{(\xi-s)^{\alpha-1}}{\Gamma(\alpha)} p(s) f_{n, y}(s) \mathrm{d} s .
\end{aligned}
$$

It is easy to show that $T_{1} y \in X$. So $T_{1}: Y \rightarrow X$ is well defined.

Step 2 Suppose that $\alpha-1<m<\alpha$. Prove that $T_{1}$ is continuous.

Let $\left\{y_{i} \in Y\right\}$ be a sequence such that $y_{i} \rightarrow y_{0}$ as $i \rightarrow$ $+\infty$ in $Y$. Then there exists $r>0$ such that

$$
\max \left\{\sup _{t \in(0,1]} t^{2-\beta}\left|y_{i}(t)\right|, \sup _{t \in(0,1]} t^{2+n-\beta}\left|D_{0^{+}}^{n} y_{i}(t)\right|\right\} \leq r
$$

holds for $i=0,1,2, \ldots$.

Then (iii) and (iv) imply that there exists a number $M_{r}>0$ and $\phi_{0}, \psi_{0} \in L^{1}(0,1)$ such that

$$
\begin{aligned}
\mid f\left(t, y_{i}(t), D_{0^{+}}^{n} y_{i}(t) \mid=\right. & \mid f\left(t, t^{\beta-2} t^{2-\beta} y_{i}(t),\right. \\
& \left.t^{\beta-n-2} t^{2+n-\beta} D_{0^{+}}^{n} y_{i}(t)\right) \mid \leq M_{r}, \\
\mid \phi_{1}\left(t, y_{i}(t), D_{0^{+}}^{n} y_{i}(t) \mid \leq\right. & \phi_{0}(t), \\
\mid \psi_{1}\left(t, y_{i}(t), D_{0^{+}}^{n} y_{i}(t) \mid \leq\right. & \psi_{0}(t)
\end{aligned}
$$

for all $t \in(0,1)$. By a direct computation, we get $\left(T_{1} y_{i}\right)(t)$ and $D_{0^{+}}^{m}\left(T_{1} y_{i}\right)(t)$. One sees that 


$$
\begin{aligned}
& t^{2-\alpha} \mid \int_{0}^{t} \frac{(t-s)^{\alpha-1}}{\Gamma(\alpha)} p(s) f_{n, y_{i}}(s) \mathrm{d} s-\int_{0}^{t} \frac{(t-s)^{\alpha-1}}{\Gamma(\alpha)} p(s) f_{n, y_{i}}(s) \mathrm{d} s \mid \\
& \leq 2 t^{2-\alpha} \int_{0}^{t} \frac{(t-s)^{\alpha-1}}{\Gamma(\alpha)} s^{k_{1}}(1-s)^{l_{1}} M_{r} \mathrm{~d} s \\
&= \frac{2 M_{r} \mathbf{B}\left(\alpha+l_{1}, k_{1}+1\right)}{\Gamma(\alpha)} t^{2+k_{1}+l_{1}} \leq \frac{2 M_{r} \mathbf{B}\left(\alpha+l_{1}, k_{1}+1\right)}{\Gamma(\alpha)} \\
& t^{2+m-\alpha} \mid \int_{0}^{t} \frac{(t-s)^{\alpha-m-1}}{\Gamma(\alpha-m)} p(s) f_{n, y_{i}}(s) \mathrm{d} s \\
& \quad-\int_{0}^{t} \frac{(t-s)^{\alpha-m-1}}{\Gamma(\alpha-m)} p(s) f_{n, y_{i}}(s) \mathrm{d} s \mid \\
& \leq 2 M_{r} t^{2+m-\alpha} \int_{0}^{t} \frac{(t-s)^{\alpha-m-1}}{\Gamma(\alpha-m)} s^{k_{1}}(1-s)^{l_{1}} \mathrm{~d} s \\
&= 2 M_{r} t^{2+m-\alpha} t^{\alpha-m+k_{1}+l_{1}} \int_{0}^{1} \frac{(1-w)^{\alpha-m+l_{1}-1}}{\Gamma(\alpha-m)} w^{k_{1}} \mathrm{~d} s \\
& \leq \frac{2 M_{r} \mathbf{B}\left(\alpha-m+l_{1}, k_{1}+1\right)}{\Gamma(\alpha-m)} t^{2+k_{1}+l_{1}} \\
& \leq \frac{2 M_{r} \mathbf{B}\left(\alpha-m+l_{1}, k_{1}+1\right)}{\Gamma(\alpha-m)} .
\end{aligned}
$$

We can show using the dominant convergence theorem that $T_{1} y_{i} \rightarrow T_{1} y_{0}$ as $i \rightarrow+\infty$. Then $T_{1}$ is continuous.

Now we prove that $T_{1}$ maps bounded sets in $Y$ into relatively compact sets in $X$. Let $\Omega \subset Y$ be a bounded subset. Then there exists $r>0$ such that

$\max \left\{\sup _{t \in(0,1]} t^{2-\beta}|y(t)|, \sup _{t \in(0,1]} t^{2+n-\beta}\left|D_{0^{+}}^{n} y(t)\right|\right\} \leq r$

holds for all $y \in \Omega$. Then (iii) and (iv) imply that there exists a number $M_{r}>0$ and $\phi_{0}, \psi_{0} \in L^{1}(0,1)$ such that

$$
\begin{gathered}
\mid f\left(t, y(t), D_{0^{+}}^{n} y(t)|=| f\left(t, t^{\beta-2} t^{2-\beta} y(t),\right.\right. \\
\left.\quad t^{\beta-n-2} t^{2+n-\beta} D_{0^{+}}^{n} y(t)\right) \mid \leq M_{r}, \\
\mid \phi_{1}\left(t, y(t), D_{0^{+}}^{n} y(t)\left|\leq \phi_{0}(t), \quad\right| \psi_{1}\left(t, y(t), D_{0^{+}}^{n} y(t) \mid \leq \psi_{0}(t)\right.\right.
\end{gathered}
$$

for all $t \in(0,1)$.

Step 3 Suppose that $\alpha-1<m<\alpha$. Prove that $\left\{T_{1} y: y \in\right.$ $\Omega\}$ is a bounded set in $X$.

Similar to Step 1 and Step 2, we can show that

$$
\begin{aligned}
& t^{2-\alpha}\left|\left(T_{1} y\right)(t)\right| \\
& \leq \frac{\left|1-a \xi^{\alpha-2}\right|+|a| \xi^{\alpha-1}}{|\Delta|} \int_{0}^{1} \psi_{0}(s) \mathrm{d} s \\
& +\frac{\left|1-b \eta^{\alpha-1}\right|+\left|1-b \eta^{\alpha-2}\right|}{|\Delta|} \int_{0}^{1} \phi_{0}(s) \mathrm{d} s \\
& +\frac{M_{r} \mathbf{B}\left(\alpha+l_{1}, k_{1}+1\right)}{\Gamma(\alpha)}+\frac{\left|1-a \xi^{\alpha-2}\right|+|a| \xi^{\alpha-1}}{|\Delta|} \\
& \times \frac{2 M_{r} \mathbf{B}\left(\alpha+l_{1}, k_{1}+1\right)}{\Gamma(\alpha)} \\
& +\frac{\left|b\left(1-a \xi^{\alpha-2}\right)\right|+|a b| \xi^{\alpha-1}}{|\Delta|} \eta^{-2-k_{1}-l_{1}} \frac{M_{r} \mathbf{B}\left(\alpha+l_{1}, k_{1}+1\right)}{\Gamma(\alpha)} \\
& +\frac{\left|a\left(1-b \eta^{\alpha-2}\right)\right|+\left|a\left(1-b \eta^{\alpha-1}\right)\right|}{|\Delta|} \\
& \xi^{-2-k_{1}-l_{1}} \frac{M_{r} \mathbf{B}\left(\alpha+l_{1}, k_{1}+1\right)}{\Gamma(\alpha)}, \\
& t^{2+m-\alpha} D_{0^{+}}^{m}\left(T_{1} y\right)(t) \\
& \leq \frac{\left|1-a \xi^{\alpha-2}\right| \frac{\Gamma(\alpha)}{\Gamma(\alpha-m)}+|a| \xi^{\alpha-1} \frac{\Gamma(\alpha-1)}{|\Gamma(\alpha-m-1)|}}{|\Delta|} \\
& \times \int_{0}^{1} \psi_{0}(s) \mathrm{d} s \\
& +\frac{\left|1-b \eta^{\alpha-1}\right| \frac{\Gamma(\alpha-1)}{|\Gamma(\alpha-m-1)|}+\left|1-b \eta^{\alpha-2}\right| \frac{\Gamma(\alpha)}{\Gamma(\alpha-m)}}{|\Delta|} \int_{0}^{1} \phi_{0}(s) \mathrm{d} s \\
& +\frac{M_{r} \mathbf{B}\left(\alpha-m+l_{1}, k_{1}+1\right)}{\Gamma(\alpha-m)} \\
& +\frac{\left|1-a \xi^{\alpha-2}\right| \frac{\Gamma(\alpha)}{\Gamma(\alpha-m)}+|a| \xi^{\alpha-1} \frac{\Gamma(\alpha-1)}{|\Gamma(\alpha-m-1)|}}{|\Delta|} \\
& \times \frac{M_{r} \mathbf{B}\left(\alpha-m+l_{1}, k_{1}+1\right)}{\Gamma(\alpha-m)} \\
& +\frac{\left|b\left(1-a \xi^{\alpha-2}\right)\right| \frac{\Gamma(\alpha)}{\Gamma(\alpha-m)}+|a b| \xi^{\alpha-1} \frac{\Gamma(\alpha-1)}{|\Gamma(\alpha-m-1)|}}{|\Delta|} \eta^{m-2-k_{1}-l_{1}} \\
& \times \frac{M_{r} \mathbf{B}\left(\alpha-m+l_{1}, k_{1}+1\right)}{\Gamma(\alpha-m)} \\
& +\frac{\left|a\left(1-b \eta^{\alpha-2}\right)\right| \frac{\Gamma(\alpha)}{\Gamma(\alpha-m)}+\left|a\left(1-b \eta^{\alpha-1}\right)\right| \frac{\Gamma(\alpha-1)}{|\Gamma(\alpha-m-1)|}}{|\Delta|} \\
& \times \xi^{m-2-k_{1}-l_{1}} \frac{M_{r} \mathbf{B}\left(\alpha-m+l_{1}, k_{1}+1\right)}{\Gamma(\alpha-m)} .
\end{aligned}
$$

So $T_{1}$ maps bounded sets into bounded sets in $X$. 
Step 4 Suppose that $\alpha-1<m<\alpha$. Prove that $\left\{T_{1} y: y \in\right.$ $\Omega\}$ is a relatively compact set in $X$.

We prove first that both $\left\{t^{2-\alpha}\left(T_{1} y\right)(t): y \in \Omega\right\}$ and $\left\{t^{2+m-\alpha} D_{0^{+}}^{m}\left(T_{1} y\right)(t): y \in \Omega\right\}$ are equi-continuous on $(0,1]$. By the definition of $T_{1}$, it suffices to show that both

$$
\begin{aligned}
& \left\{t^{2-\alpha} \int_{0}^{t}(t-s)^{\alpha-1} p(s) f_{n, y}(s) \mathrm{d} s: y \in \Omega\right\} \text { and } \\
& \left\{t^{2+m-\alpha} \int_{0}^{t}(t-s)^{\alpha-m-1} p(s) f_{n, y}(s) \mathrm{d} s: y \in \Omega\right\}
\end{aligned}
$$

are equi-continuous on $(0,1]$ (we can prove that the other parts of $\left\{t^{2-\alpha}\left(T_{1} y\right)(t): y \in \Omega\right\}$ and $\left\{t^{2+m-\alpha} D_{0^{+}}^{m}\left(T_{1} y\right)(t)\right.$ : $y \in \Omega\}$ are equi-continuous on $(0,1]$ similar to [1]). Then, we prove that both $\left\{t^{2-\alpha}\left(T_{1} y\right)(t): y \in \Omega\right\}$ and $\left\{t^{2+m-\alpha} D_{0^{+}}^{n}\left(T_{1} y\right)(t): y \in \Omega\right\}$ are equi-convergent as $t \rightarrow 0$. By the definition of $T_{1}$, it suffices to show that both

$$
\begin{aligned}
& \left\{t^{2-\alpha} \int_{0}^{t}(t-s)^{\alpha-1} p(s) f_{n, y}(s) \mathrm{d} s: y \in \Omega\right\} \text { and } \\
& \left\{t^{2+m-\alpha} \int_{0}^{t}(t-s)^{\alpha-m-1} p(s) f_{n, y}(s) \mathrm{d} s: y \in \Omega\right\}
\end{aligned}
$$

are equi-convergent as $t \rightarrow 0$.

First, let $t_{1}, t_{2} \in[e, f] \subset(0,1]$ with $t_{1}<t_{2}, 0<e<f \leq 1$, and $y \in \Omega$. Then we have

$$
\begin{aligned}
& \mid t_{1}^{2-\alpha} \int_{0}^{t_{1}}\left(t_{1}-s\right)^{\alpha-1} p(s) f_{n, y}(s) \mathrm{d} s-t_{2}^{2-\alpha} \\
& \int_{0}^{t_{2}}\left(t_{2}-s\right)^{\alpha-1} p(s) f_{n, y}(s) \mathrm{d} s \\
& \leq\left|t_{1}^{2-\alpha}-t_{2}^{2-\alpha}\right| \int_{0}^{t_{2}}\left(t_{2}-s\right)^{\alpha-1}\left|p(s) f_{n, y}(s)\right| \mathrm{d} s \\
& +t_{1}^{2-\alpha} \int_{t_{1}}^{t_{2}}\left(t_{2}-s\right)^{\alpha-1}\left|p(s) f_{n, y}(s)\right| \mathrm{d} s \\
& +t_{1}^{2-\alpha} \int_{0}^{t_{1}}\left|\left(t_{1}-s\right)^{\alpha-1}-\left(t_{2}-s\right)^{\alpha-1}\right|\left|p(s) f_{n, y}(s)\right| \mathrm{d} s \\
& \leq M_{r}\left|t_{1}^{2-\alpha}-t_{2}^{2-\alpha}\right| \int_{0}^{t_{2}}\left(t_{2}-s\right)^{\alpha-1} s^{k_{1}}(1-s)^{l_{1}} \mathrm{~d} s+M_{r} t_{1}^{2-\alpha} \\
& \int_{t_{1}}^{t_{2}}\left(t_{2}-s\right)^{\alpha-1} s^{k_{1}}(1-s)^{l_{1}} \mathrm{~d} s \\
& +M_{r} t_{1}^{2-\alpha} \int_{0}^{t_{1}}\left|\left(t_{1}-s\right)^{\alpha-1}-\left(t_{2}-s\right)^{\alpha-1}\right| s^{k_{1}}(1-s)^{l_{1}} \mathrm{~d} s \\
& \leq M_{r}\left|t_{1}^{2-\alpha}-t_{2}^{2-\alpha}\right| t_{2}^{\alpha+k_{1}+l_{1}} \mathbf{B}\left(\alpha+l_{1}, k_{1}+1\right)+M_{r} t_{1}^{2-\alpha} t_{2}^{\alpha+l_{1}+k_{1}} \\
& \int_{\frac{t_{1}}{t_{2}}}^{1}(1-w)^{\alpha+l_{1}-1} w^{k_{1}} \mathrm{~d} w \\
& +M_{r} t_{2}^{2-\alpha} \int_{0}^{1}\left[\left(t_{2}-s\right)^{\alpha-1}-\left(t_{1}-s\right)^{\alpha-1}\right] s^{k_{1}}(1-s)^{l_{1}} \mathrm{~d} s
\end{aligned}
$$

$\rightarrow 0$ uniformly in $\Omega$ as $t_{1} \rightarrow t_{2}$ on $[e, f]$.
Second, let $t_{1}, t_{2} \in[e, f] \subset(0,1]$ with $0<e \leq t_{1}<t_{2} \leq f \leq 1$ and $y \in \Omega$. Then we have

$\mid t_{1}^{2+m-\alpha} \int_{0}^{t_{1}}\left(t_{1}-s\right)^{\alpha-m-1} p(s) f_{n, y}(s) \mathrm{d} s-t_{2}^{2+m-\alpha}$

$$
\begin{aligned}
& \int_{0}^{t_{2}}\left(t_{2}-s\right)^{\alpha-m-1} p(s) f_{n, y}(s) \mathrm{d} s \\
& \leq\left|t_{1}^{2+m-\alpha}-t_{2}^{2+m-\alpha}\right| \int_{0}^{t_{2}}\left(t_{2}-s\right)^{\alpha-m-1}\left|p(s) f_{n, y}(s)\right| \mathrm{d} s \\
& +t_{1}^{2+m-\alpha} \int_{t_{1}}^{t_{2}}\left(t_{2}-s\right)^{\alpha-m-1}\left|p(s) f_{n, y}(s)\right| \mathrm{d} s \\
& +t_{1}^{2+m-\alpha} \int_{0}^{t_{1}}\left|\left(t_{1}-s\right)^{\alpha-m-1}-\left(t_{2}-s\right)^{\alpha-m-1}\right|\left|p(s) f_{n, y}(s)\right| \mathrm{d} s \\
& \leq M_{r}\left|t_{1}^{2+m-\alpha}-t_{2}^{2+m-\alpha}\right| \int_{0}^{t_{2}}\left(t_{2}-s\right)^{\alpha-m-1} s^{k_{1}}(1-s)^{l_{1}} \mathrm{~d} s \\
& +M_{r} t_{1}^{2+m-\alpha} \int_{t_{1}}^{t_{2}}\left(t_{2}-s\right)^{\alpha-m-1} s^{k_{1}}(1-s)^{l_{1}} \mathrm{~d} s \\
& +M_{r} t_{1}^{2+m-\alpha} \int_{0}^{t_{1}}\left[\left(t_{1}-s\right)^{\alpha-m-1}-\left(t_{2}-s\right)^{\alpha-m-1}\right] s^{k_{1}}(1-s)^{l_{1}} \mathrm{~d} s \\
& \leq M_{r}\left|t_{1}^{2+m-\alpha}-t_{2}^{2+m-\alpha}\right| t_{2}^{\alpha-m+k_{1}+l_{1}} \mathbf{B}\left(\alpha-m+l_{1}, k_{1}+1\right) \\
& +M_{r} t_{1}^{2+m-\alpha} t_{2}^{\alpha-m+k_{1}+l_{1}} \int_{\frac{t_{1}}{t_{2}}}^{1}(1-w)^{\alpha-m-1+l_{1}} w^{k_{1}} \mathrm{~d} w \\
& +M_{r} t_{1}^{2+m-\alpha} \int_{0}^{t_{1}}\left(t_{1}-s\right)^{\alpha-m-1} s^{k_{1}}\left(t_{1}-s\right)^{l_{1}} \mathrm{~d} s \\
& -M_{r} t_{1}^{2+m-\alpha} \int_{0}^{t_{1}}\left(t_{2}-s\right)^{\alpha-m-1} s^{k_{1}}\left(t_{2}-s\right)^{l_{1}} \mathrm{~d} s \\
& \leq M_{r}\left|t_{1}^{2+m-\alpha}-t_{2}^{2+m-\alpha}\right| t_{2}^{\alpha-m+k_{1}+l_{1}} \mathbf{B}\left(\alpha-m+l_{1}, k_{1}+1\right) \\
& +M_{r} \max \left\{e^{\alpha-m+k_{1}+l_{1}}, f^{\alpha-m+k_{1}+l_{1}}\right\} \int_{\frac{t_{1}}{t_{2}}}^{1}(1-w)^{\alpha-m-1+l_{1}} w^{k_{1}} \mathrm{~d} w \\
& +M_{r} t_{1}^{2+m-\alpha} t_{1}^{\alpha-m+k_{1}+l_{1}} \int_{0}^{1}(1-w)^{\alpha-m+l_{1}-1} w^{k_{1}} \mathrm{~d} w \\
& -M_{r} t_{1}^{2+m-\alpha} t_{2}^{\alpha-m+k_{1}+l_{1}} \int_{0}^{\frac{t_{1}}{t_{2}}}(1-w)^{\alpha-m+l_{1}-1} w^{k_{1}} \mathrm{~d} w \\
& \leq M_{r}\left|t_{1}^{2+m-\alpha}-t_{2}^{2+m-\alpha}\right| t_{2}^{\alpha-m+k_{1}+l_{1}} \mathbf{B}\left(\alpha-m+l_{1}, k_{1}+1\right) \\
& +M_{r} \max \left\{e^{\alpha-m+k_{1}+l_{1}}, f^{\alpha-m+k_{1}+l_{1}}\right\} \int_{{\frac{t_{1}}{t_{2}}}}^{1}(1-w)^{\alpha-m-1+l_{1}} w^{k_{1}} \mathrm{~d} w \\
& +M_{r} t_{1}^{2+m-\alpha}\left(t_{1}^{\alpha-m+k_{1}+l_{1}}-t_{2}^{\alpha-m+k_{1}+l_{1}}\right) \int_{0}^{1}(1-w)^{\alpha-m+l_{1}-1} w^{k_{1}} \mathrm{~d} w \\
& +M_{r} t_{1}^{2+m-\alpha} t_{2}^{\alpha-m+k_{1}+l_{1}} \int_{\frac{t_{1}}{t_{2}}}^{1}(1-w)^{\alpha-m+l_{1}-1} w^{k_{1}} \mathrm{~d} w
\end{aligned}
$$

$\rightarrow 0$ uniformly in $\Omega$ as $t_{1} \rightarrow t_{2}$ on $[e, f]$. 
Third, we have

$$
\begin{aligned}
& \mid t^{2-\alpha}\left(T_{1} y\right)(t)-\left(\frac{a \xi^{\alpha-1}}{\Delta} \int_{0}^{1} \psi_{1 n, y}(s) \mathrm{d} s+\frac{\left(1-b \eta^{\alpha-1}\right)}{\Delta}\right. \\
& \times \int_{0}^{1} \phi_{1 n, y}(s) \mathrm{d} s . \\
& +\frac{a \xi^{\alpha-1}}{\Delta} \int_{0}^{1} \frac{(1-s)^{\alpha-1}}{\Gamma(\alpha)} p(s) f_{n, y}(s) \mathrm{d} s-\frac{a b \xi^{\alpha-1}}{\Delta} \\
& \times \int_{0}^{\eta} \frac{(\eta-s)^{\alpha-1}}{\Gamma(\alpha)} p(s) f_{n, x}(s) \mathrm{d} s \\
& \left.-\frac{a\left(1-b \eta^{\alpha-1}\right)}{\Delta} \int_{0}^{\xi} \frac{(\xi-s)^{\alpha-1}}{\Gamma(\alpha)} p(s) f_{n, y}(s) \mathrm{d} s\right) \\
& \leq \frac{\left|1-a \xi^{\alpha-2}\right| t}{|\Delta|} \int_{0}^{1} \psi_{0} \mathrm{~d} s+\frac{\left|1-b \eta^{\alpha-2}\right| t}{|\Delta|} \int_{0}^{1} \phi_{0}(s) \mathrm{d} s \\
& +t^{2-\alpha} \int_{0}^{t} \frac{(t-s)^{\alpha-1}}{\Gamma(\alpha)}\left|p(s) f_{n, y}(s)\right| \mathrm{d} s+\frac{\left|1-a \xi^{\alpha-2}\right| t}{|\Delta|} \\
& \times \int_{0}^{1} \frac{(1-s)^{\alpha-1}}{\Gamma(\alpha)}\left|p(s) f_{n, y}(s)\right| \mathrm{d} s \\
& +\frac{\left|b\left(1-a \xi^{\alpha-2}\right)\right| t}{|\Delta|} \int_{0}^{\eta} \frac{(\eta-s)^{\alpha-1}}{\Gamma(\alpha)}\left|p(s) f_{n, x}(s)\right| \mathrm{d} s \\
& +\frac{\left|a\left(1-b \eta^{\alpha-2}\right)\right| t}{|\Delta|} \int_{0}^{\xi} \frac{(\xi-s)^{\alpha-1}}{\Gamma(\alpha)}\left|p(s) f_{n, y}(s)\right| \mathrm{d} s \\
& \leq \frac{\left|1-a \xi^{\alpha-2}\right| t}{|\Delta|} \int_{0}^{1} \psi_{0} \mathrm{~d} s+\frac{\left|1-b \eta^{\alpha-2}\right| t}{|\Delta|} \int_{0}^{1} \phi_{0}(s) \mathrm{d} s \\
& +t^{2+k_{1}+l_{1}} \frac{\mathbf{B}\left(\alpha+l_{1}, k_{1}+1\right)}{\Gamma(\alpha)} M_{r}+\frac{\left|1-a \xi^{\alpha-2}\right| t}{|\Delta|} \\
& \times \frac{\mathbf{B}\left(\alpha+l_{1}, k_{1}+1\right)}{\Gamma(\alpha)} M_{r} \\
& +\frac{\left|b\left(1-a \xi^{\alpha-2}\right)\right| t}{|\Delta|} \frac{\mathbf{B}\left(\alpha+l_{1}, k_{1}+1\right)}{\Gamma(\alpha)} M_{r} \\
& +\frac{\left|a\left(1-b \eta^{\alpha-2}\right)\right| t}{|\Delta|} \frac{\mathbf{B}\left(\alpha+l_{1}, k_{1}+1\right)}{\Gamma(\alpha)} M_{r}
\end{aligned}
$$

Fourth, we have

$$
\begin{gathered}
\mid t^{2+m-\alpha} D_{0^{+}}^{m}\left(T_{1} y\right)(t)-\left(\frac{a \xi^{\alpha-1} \frac{\Gamma(\alpha-1)}{\Gamma(\alpha-m-1)}}{\Delta} \int_{0}^{1} \psi_{1 n, y}(s) \mathrm{d} s\right. \\
+\frac{\left(1-b \eta^{\alpha-1}\right) \frac{\Gamma(\alpha-1)}{\Gamma(\alpha-m-1)}}{\Delta} \int_{0}^{1} \phi_{1 n, y}(s) \mathrm{d} s \\
+\frac{a \xi^{\alpha-1} \frac{\Gamma(\alpha-1)}{\Gamma(\alpha-m-1)}}{\Delta}
\end{gathered}
$$

$$
\begin{aligned}
& \times \int_{0}^{1} \frac{(1-s)^{\alpha-1}}{\Gamma(\alpha)} p(s) f_{n, y}(s) \mathrm{d} s \\
& -\frac{a b \xi^{\alpha-1} \frac{\Gamma(\alpha-1)}{\Gamma(\alpha-m-1)}}{\Delta} \int_{0}^{\eta} \frac{(\eta-s)^{\alpha-1}}{\Gamma(\alpha)} p(s) f_{n, x}(s) \mathrm{d} s \\
& \left.-\frac{a\left(1-b \eta^{\alpha-1}\right) \frac{\Gamma(\alpha-1)}{\Gamma(\alpha-m-1)}}{\Delta} \int_{0}^{\xi} \frac{(\xi-s)^{\alpha-1}}{\Gamma(\alpha)} p(s) f_{n, y}(s) \mathrm{d} s\right) \\
& \leq \frac{\left|1-a \xi^{\alpha-2}\right| \frac{\Gamma(\alpha)}{\Gamma(\alpha-m)} t}{|\Delta|} \int_{0}^{1} \psi_{0}(s) \mathrm{d} s+\frac{\left|1-b \eta^{\alpha-2}\right| \frac{\Gamma(\alpha)}{\Gamma(\alpha-m)} t}{|\Delta|} \\
& \times \int_{0}^{1} \phi_{0}(s) \mathrm{d} s \\
& +t^{2+m-\alpha} \int_{0}^{t} \frac{(t-s)^{\alpha-m-1}}{\Gamma(\alpha-m)} s^{k_{1}}(1-s)^{l_{1}} M_{r} \mathrm{~d} s \\
& +\frac{\left|1-a \xi^{\alpha-2}\right| \frac{\Gamma(\alpha)}{\Gamma(\alpha-m)} t}{|\Delta|} \int_{0}^{1} \frac{(1-s)^{\alpha-1}}{\Gamma(\alpha)} s^{k_{1}}(1-s)^{l_{1}} M_{r} \mathrm{~d} s \\
& +\frac{\left|b\left(1-a \xi^{\alpha-2}\right)\right| \frac{\Gamma(\alpha)}{\Gamma(\alpha-m)} t}{|\Delta|} \int_{0}^{\eta} \frac{(\eta-s)^{\alpha-1}}{\Gamma(\alpha)} s^{k_{1}}(1-s)^{l_{1}} M_{r} \mathrm{~d} s \\
& +\frac{\left|a\left(1-b \eta^{\alpha-2}\right)\right| \frac{\Gamma(\alpha)}{\Gamma(\alpha-m)} t}{|\Delta|} \int_{0}^{\xi} \frac{(\xi-s)^{\alpha-1}}{\Gamma(\alpha)} s^{k_{1}}(1-s)^{l_{1}} M_{r} \mathrm{~d} s \\
& \leq \frac{\left|1-a \xi^{\alpha-2}\right| \frac{\Gamma(\alpha)}{\Gamma(\alpha-m)} t}{|\Delta|} \int_{0}^{1} \psi_{0}(s) \mathrm{d} s+\frac{\left|1-b \eta^{\alpha-2}\right| \frac{\Gamma(\alpha)}{\Gamma(\alpha-m)} t}{|\Delta|} \\
& \times \int_{0}^{1} \phi_{0}(s) \mathrm{d} s \\
& +t^{2+k_{1}+l_{1}} \frac{\mathbf{B}\left(\alpha-m+l_{1}, k_{1}+1\right)}{\Gamma(\alpha-m)} M_{r} \\
& +\frac{\left|1-a \xi^{\alpha-2}\right| \frac{\Gamma(\alpha)}{\Gamma(\alpha-m)} t}{|\Delta|} \frac{\mathbf{B}\left(\alpha+l_{1}, k_{1}+1\right)}{\Gamma(\alpha)} M_{r} \\
& +\frac{\left|b\left(1-a \xi^{\alpha-2}\right)\right| \frac{\Gamma(\alpha)}{\Gamma(\alpha-m)} t}{|\Delta|} \eta^{\alpha-2} \frac{\mathbf{B}\left(\alpha+l_{1}, k_{1}+1\right)}{\Gamma(\alpha)} M_{r} \\
& +\frac{\left|a\left(1-b \eta^{\alpha-2}\right)\right| \frac{\Gamma(\alpha)}{\Gamma(\alpha-m)} t}{|\Delta|} \xi^{\alpha-2} \frac{\mathbf{B}\left(\alpha+l_{1}, k_{1}+1\right)}{\Gamma(\alpha)} M_{r} \\
& \rightarrow 0 \text { uniformly on } \Omega \text { as } t \rightarrow 0 \text {. }
\end{aligned}
$$

Therefore, $T_{1} \Omega$ is relatively compact.

From above discussion, $T_{1}$ is completely continuous. The proof is completed. 
Define

$$
G(t, s)=\frac{1}{\Gamma(\alpha) \Delta}\left\{\begin{array}{l}
\left(v_{1} t^{\alpha-1}+\mu_{1} t^{\alpha-2}\right)(1-s)^{\alpha-1} \\
+\left(\lambda_{1} a t^{\alpha-1}-\omega_{1} a t^{\alpha-2}\right)(\xi-s)^{\alpha-1} \\
-\left(v_{1} b t^{\alpha-1}+b \mu_{1} t^{\alpha-2}\right)(\eta-s)^{\alpha-1} \\
-\left(\mu_{1} \lambda_{1}+\omega_{1} v_{1}\right)(t-s)^{\alpha-1}, \\
\left(v_{1} t^{\alpha-1}+\mu_{1} t^{\alpha-2}\right)(1-s)^{\alpha-1} \\
-\left(v_{1} b t^{\alpha-1}+b \mu_{1} t^{\alpha-2}\right)(\eta-s)^{\alpha-1} \xi<s \leq \min \{t, \xi\}, \eta t, \\
-\left(\mu_{1} \lambda_{1}+\omega_{1} v_{1}\right)(t-s)^{\alpha-1}, \\
\left(v_{1} t^{\alpha-1}+\mu_{1} t^{\alpha-2}\right)(1-s)^{\alpha-1} \\
-\left(v_{1} b t^{\alpha-1}+b \mu_{1} t^{\alpha-2}\right)(\eta-s)^{\alpha-1}, \\
\left(v_{1} t^{\alpha-1}+\mu_{1} t^{\alpha-2}\right)(1-s)^{\alpha-1} \\
\left.+\left(\lambda_{1} a t^{\alpha-1}-\omega_{1} a t^{\alpha-2}\right)(\xi-s)^{\alpha-1} t<s \leq \xi\right\}<s \leq \eta, \\
-\left(v_{1} b t^{\alpha-1}+b \mu_{1} t^{\alpha-2}\right)(\eta-s)^{\alpha-1}, \\
\left(v_{1} t^{\alpha-1}+\mu_{1} t^{\alpha-2}\right)(1-s)^{\alpha-1} \\
-\left(\mu_{1} \lambda_{1}+\omega_{1} v_{1}\right)(t-s)^{\alpha-1}, \eta<s \leq t, \\
\left(v_{1} t^{\alpha-1}+\mu_{1} t^{\alpha-2}\right)(1-s)^{\alpha-1}, \max \{\eta, t\}<s \leq 1,
\end{array}\right.
$$

and

$H(t, s)=\frac{1}{\Gamma(\beta) \nabla}\left\{\begin{array}{l}\left(v_{2} t^{\beta-1}+\mu_{2} t^{\beta-2}\right)(1-s)^{\beta-1} \\ +\left(\lambda_{2} c t^{\beta-1}-\omega_{2} c t^{\beta-2}\right)(\xi-s)^{\beta-1} 0 \leq s \leq \min \{t, \xi\}, \\ -\left(\mu_{2} \lambda_{2}+\omega_{2} v_{2}\right)(t-s)^{\beta-1}, \\ \left(v_{2} t^{\beta-1}+\mu_{2} t^{\beta-2}\right)(1-s)^{\beta-1} \\ -\left(v_{2} \mathrm{~d} t^{\beta-1}+d \mu_{2} t^{\beta-2}\right)(\eta-s)^{\beta-1} \xi<s \leq \min \{t, \eta\}, \\ -\left(\mu_{2} \lambda_{2}+\omega_{2} v_{2}\right)(t-s)^{\beta-1}, \\ \left(v_{2} t^{\beta-1}+\mu_{2} t^{\beta-2}\right)(1-s)^{\beta-1} \\ -\left(v_{2} \mathrm{~d} t^{\beta-1}+d \mu_{2} t^{\beta-2}\right)(\eta-s)^{\beta-1}, \max \{t, \xi\}<s \leq \eta, \\ \left(v_{2} t^{\beta-1}+\mu_{2} t^{\beta-2}\right)(1-s)^{\beta-1} \\ +\left(\lambda_{2} c t^{\beta-1}-\omega_{2} c t^{\beta-2}\right)(\xi-s)^{\beta-1} t<s \leq \xi \\ -\left(v_{2} \mathrm{~d} t^{\beta-1}+d \mu_{2} t^{\beta-2}\right)(\eta-s)^{\beta-1}, \\ \left(v_{2} t^{\beta-1}+\mu_{2} t^{\beta-2}\right)(1-s)^{\beta-1} \\ -\left(\mu_{2} \lambda_{2}+\omega_{2} v_{2}\right)(t-s)^{\beta-1}, \eta<\leq t, \\ \left(v_{2} t^{\beta-1}+\mu_{2} t^{\beta-2}\right)(1-s)^{\beta-1}, \max \{\eta, t\}<s \leq 1 .\end{array}\right.$

Now, we rewrite

$$
\begin{aligned}
(T(x, y))(t)= & \left(\left(T_{1} y\right)(t),\left(T_{2} x\right)(t)\right) \\
= & \left(\frac{v_{1} t^{\alpha-1}+\mu_{1} t^{\alpha-2}}{\Delta} \int_{0}^{1} \psi_{1 n, y}(s) \mathrm{d} s\right. \\
& +\frac{\omega_{1} t^{\alpha-2}-\lambda_{1} t^{\alpha-1}}{\Delta} \int_{0}^{1} \phi_{1 n, y}(s) \mathrm{d} s \\
& +\int_{0}^{1} G(t, s) p(s) f_{n, y}(s) \mathrm{d} s, \\
& \frac{v_{2} t^{\beta-1}+\mu_{2} t^{\beta-2}}{\nabla} \int_{0}^{1} \psi_{2 m, x}(s) \mathrm{d} s \\
& +\frac{\omega_{2} t^{\beta-2}-\lambda_{2} t^{\beta-1}}{\nabla} \int_{0}^{1} \phi_{2 m, x}(s) \mathrm{d} s \\
& \left.+\int_{0}^{1} H(t, s) g_{m, x}(s) \mathrm{d} s\right) .
\end{aligned}
$$

Lemma 2.5 (Lemma 2.9 in [11]) Suppose that $a, b, c, d \geq 0$, and

$$
\begin{aligned}
& \Delta>0, \quad 0 \leq a<\frac{1}{\xi^{\alpha-2}(1-\xi)}, \quad 0 \leq b<\frac{1}{\eta^{\alpha-1}}, \\
& \nabla>0, \quad 0 \leq c<\frac{1}{\xi^{\beta-2}(1-\xi)}, \quad 0 \leq d<\frac{1}{\eta^{\beta-1}} .
\end{aligned}
$$

\section{Then}

$G(t, s) \geq 0$ for all $t, s \in(0,1), H(t, s) \geq 0$ for all $t, s \in(0,1)$.

\section{Main results}

In this section, we prove existence result on solutions of $\operatorname{BVP}(1)$. Let $\mu_{i}, v_{i}, \omega_{i}, \lambda_{i}(i=1,2)$ and $\Delta, \nabla$ be defined by (10). For $\Phi \in L^{1}(0,1)$, denote $\|\Phi\|_{1}=\int_{0}^{1}|\Phi(s)| \mathrm{d} s$. The following assumption will be used in the main theorem.

A function $\Phi:[0, \infty) \times[0, \infty) \rightarrow[0, \infty)$ is called a biincreasing function if both $u \rightarrow \Phi(u, v)$ and $v \rightarrow \Phi(u, v)$ are increasing. We now list the following assumption:

(B1) there exist $\bar{\phi}_{i}, \bar{\psi}_{i} \in L^{1}(0,1)(i=1,2)$ and bi-increasing functions $\Phi, \Psi, \Phi_{i}, \Psi_{i}(i=1,2)$ such that

$$
\begin{aligned}
& \left|f\left(t, \frac{u}{t^{2-\beta}}, \frac{v}{t^{2+n-\beta}}\right)\right| \leq \Phi(|u|,|v|), t \in(0,1), u, v \in \mathbb{R}, \\
& \left|g\left(t, \frac{u}{t^{2-\alpha}}, \frac{v}{t^{2+m-\alpha}}\right)\right| \leq \Psi(|u|,|v|), t \in(0,1), u, v \in \mathbb{R}, \\
& \left|\phi_{1}\left(t, \frac{u}{t^{2-\beta}}, \frac{v}{t^{2+n-\beta}}\right)\right| \leq \bar{\phi}_{1}(t) \Phi_{1}(|u|,|v|), t \in(0,1), u, v \in \mathbb{R}, \\
& \left|\psi_{1}\left(t, \frac{u}{t^{2-\beta}}, \frac{v}{t^{2+n-\beta}}\right)\right| \leq \bar{\psi}_{1}(t) \Psi_{1}(|u|,|v|), t \in(0,1), u, v \in \mathbb{R}, \\
& \left|\phi_{2}\left(t, \frac{u}{t^{2-\alpha}}, \frac{v}{t^{2+m-\alpha}}\right)\right| \leq \bar{\phi}_{2}(t) \Phi_{2}(|u|,|v|), t \in(0,1), u, v \in \mathbb{R}, \\
& \left|\psi_{2}\left(t, \frac{u}{t^{2-\alpha}}, \frac{v}{t^{2+m-\alpha}}\right)\right| \leq \bar{\psi}_{2}(t) \Psi_{2}(|u|,|v|), t \in(0,1), u, v \in \mathbb{R} .
\end{aligned}
$$

For ease expression, denote

$$
\begin{aligned}
M_{1}= & {\left[\frac{v_{1}+\mu_{1}}{\Delta} \|+\frac{\left.v_{1} \frac{\Gamma(\alpha)}{\Gamma(\alpha-m)}+\mu_{1} \frac{\Gamma(\alpha-1)}{\Gamma(\alpha-m-1)}\right]\left\|\bar{\psi}_{1}\right\|_{1},}{\Delta}\right] } \\
N_{1}= & {\left[\frac{\omega_{1}+\lambda_{1}}{\Delta} \|+\frac{\left.\omega_{1} \frac{\Gamma(\alpha-1)}{\Gamma(\alpha-m-1)}+\lambda_{1} \frac{\Gamma(\alpha)}{\Gamma(\alpha-m)}\right]\left\|\bar{\phi}_{1}\right\|_{1},}{\Delta}\right] } \\
Q_{1}= & {\left[1+\frac{v_{1}+\mu_{1}}{\Delta}+\frac{b v_{1}+b \mu_{1}}{\Delta} \eta^{\alpha+k_{1}+l_{1}}\right.} \\
& \left.+\frac{a \lambda_{1}+a \omega_{1}}{\Delta} \xi^{\alpha+k_{1}+l_{1}}\right] \frac{\mathbf{B}\left(\alpha+l_{1}, k_{1}+1\right)}{\Gamma(\alpha)} \\
& +\frac{\mathbf{B}\left(\alpha-m+l_{1}, k_{1}+1\right)}{\Gamma(\alpha-m)}+\frac{v_{1}}{\Gamma(\alpha-m)}+\mu_{1} \frac{\Gamma(\alpha-1)}{\Gamma(\alpha-m-1) \mid} \\
& \times \frac{\mathbf{B}\left(\alpha+l_{1}, k_{1}+1\right)}{\Gamma(\alpha)} \\
& +\frac{b v_{1} \frac{\Gamma(\alpha)}{\Gamma(\alpha-m)}+b \mu_{1} \frac{\Gamma(\alpha-1)}{\Gamma(\alpha-m-1) \mid}}{\Delta} \frac{\eta^{\alpha+k_{1}+l_{1}} \mathbf{B}\left(\alpha+l_{1}, k_{1}+1\right)}{\Gamma(\alpha)} \\
& +\frac{a \lambda_{1} \frac{\Gamma(\alpha)}{\Gamma(\alpha-m)}+a \omega_{1} \frac{\Gamma(\alpha-1)}{\Gamma(\alpha-m-1) \mid}}{\Delta} \frac{\xi^{\alpha+k_{1}+l_{1}} \mathbf{B}\left(\alpha+l_{1}, k_{1}+1\right)}{\Gamma(\alpha)},
\end{aligned}
$$


and

$$
\begin{aligned}
M_{2}= & {\left[\frac{v_{2}+\mu_{2}}{\nabla} \|+\frac{\left.v_{2} \frac{\Gamma(\beta)}{\Gamma(\beta-n)}+\mu_{2} \frac{\Gamma(\beta-1)}{\Gamma(\beta-n-1)}\right]}{\nabla}\right]\left\|\bar{\psi}_{2}\right\|_{1}, } \\
N_{2}= & {\left[\frac{\omega_{2}+\lambda_{2}}{\nabla} \|+\frac{\left.\omega_{2} \frac{\Gamma(\beta-1)}{\Gamma(\beta-n-1)}+\lambda_{2} \frac{\Gamma(\beta)}{\Gamma(\beta-n)}\right]}{\nabla}\right]\left\|\bar{\phi}_{2}\right\|_{1}, } \\
Q_{2}= & {\left[1+\frac{v_{2}+\mu_{2}}{\nabla}+\frac{c v_{2}+d \mu_{2}}{\nabla} \eta^{\beta+k_{2}+l_{2}}\right.} \\
& \left.+\frac{c \lambda_{2}+c \omega_{2}}{\nabla} \xi^{\beta+k_{2}+l_{2}}\right] \frac{\mathbf{B}\left(\beta+l_{2}, k_{2}+1\right)}{\Gamma(\beta)} \\
& +\frac{\mathbf{B}\left(\beta-n+l_{2}, k_{2}+1\right)}{\Gamma(\beta-n)}+\frac{v_{2} \frac{\Gamma(\beta)}{\Gamma(\beta-n)}+\mu_{2} \frac{\Gamma(\beta-1)}{\Gamma(\beta-n-1) \mid}}{\nabla} \\
& \times \frac{\mathbf{B}\left(\beta+l_{2}, k_{2}+1\right)}{\Gamma(\beta)} \\
& +\frac{d v_{2} \frac{\Gamma(\beta)}{\Gamma(\beta-n)}+d \mu_{2} \frac{\Gamma(\beta-1)}{\Gamma(\beta-n-1))}}{\nabla} \frac{\eta^{\beta+k_{2}+l_{2}} \mathbf{B}\left(\beta+l_{2}, k_{2}+1\right)}{\Gamma(\beta)} \\
& +\frac{c \lambda_{2} \frac{\Gamma(\beta)}{\Gamma(\beta-n)}+c \omega_{2} \frac{\Gamma(\beta-1)}{\Gamma(\beta-n-1) !}}{\nabla} \frac{\xi^{\beta+k_{2}+l_{2}} \mathbf{B}\left(\beta+l_{2}, k_{2}+1\right)}{\Gamma(\beta)} .
\end{aligned}
$$

Theorem 3.1 Suppose that (12) holds, (i)-(iv) defined in Sect. 1 and (B1) hold. Then BVP(1) has at least one positive solution if

$$
\begin{aligned}
& M_{1} \Psi_{1}\left(r_{2}, r_{2}\right)+N_{1} \Phi_{1}\left(r_{2}, r_{2}\right)+Q_{1} \Phi\left(r_{2}, r_{2}\right) \leq r_{1}, \\
& M_{2} \Psi_{2}\left(r_{1}, r_{1}\right)+N_{2} \Phi_{1}\left(r_{1}, r_{1}\right)+Q_{2} \Psi\left(r_{1}, r_{1}\right) \leq r_{2}
\end{aligned}
$$

has a solution $\left(r_{1}, r_{2}\right)$ satisfying $r_{1}>0, r_{2}>0$.

Proof From Lemmas 2.2 and 2.3, we know that $(x, y)$ is a solution of BVP(1) if and only if $(x, y)$ is a fixed point of $T$. From Lemma 2.4, $T: X \times Y \rightarrow X \times Y$ is completely continuous. By Lemma 2.5 and (i)-(iv), $(x, y)$ is a positive solution if $(x, y)$ is a solution of $\operatorname{BVP}(1)$.

To get a fixed point of $T$, we apply the Schauder's fixed point theorem. We should define a closed convex bounded subset $\Omega$ of $E$ such that $T(\Omega) \subseteq \Omega$. It is easy to see that $\Omega=\left\{(x, y) \in E:\|x\| \leq r_{1},\|y\| \leq r_{2}\right\}$ is a closed convex bounded subset $\Omega$ of $E$.

For $(x, y) \in \Omega$, we get $\|x\| \leq r_{1}, \quad\|y\| \leq r_{2}$. Furthermore, we have

$$
\begin{aligned}
\left|f\left(t, y(t), D_{0^{+}}^{n} y(t)\right)\right| & \leq \Phi\left(t^{2-\beta}|y(t)|, t^{2+n-\beta}\left|D_{0^{+}}^{n} y(t)\right|\right) \\
& \leq \Phi\left(r_{2}, r_{2}\right), t \in(0,1), \\
\left|g\left(t, x(t), D_{0^{+}}^{m} x(t)\right)\right| & \leq \Psi\left(t^{2-\alpha}|x(t)|, t^{2+m-\alpha}\left|D_{0^{+}}^{m} x(t)\right|\right) \\
& \leq \Psi\left(r_{1}, r_{1}\right), t \in(0,1), \\
\left|\phi_{1}\left(t, y(t), D_{0^{+}}^{n} y(t)\right)\right| & \leq \bar{\phi}_{1}(t) \Phi_{1}\left(r_{2}, r_{2}\right), t \in(0,1), \\
\left|\psi_{1}\left(t, y(t), D_{0^{+}}^{n} y(t)\right)\right| & \leq \bar{\psi}_{1}(t) \Psi_{1}\left(r_{2}, r_{2}\right), t \in(0,1),
\end{aligned}
$$

$$
\begin{aligned}
& \left|\phi_{2}\left(t, x(t), D_{0^{+}}^{m} x(t)\right)\right| \leq \bar{\phi}_{2}(t) \Phi_{2}\left(r_{1}, r_{1}\right), t \in(0,1), \\
& \left|\psi_{2}\left(t, x(t), D_{0^{+}}^{m} x(t)\right)\right| \leq \bar{\psi}_{2}(t) \Psi_{2}\left(r_{1}, r_{1}\right), t \in(0,1) .
\end{aligned}
$$

By the definition of $T$, we have

$$
\begin{aligned}
t^{2-\alpha} \mid & \left(T_{1} y\right)(t) \mid \\
\leq & \frac{v_{1}+\mu_{1}}{\Delta}\left\|\bar{\psi}_{1}\right\|_{1} \Psi_{1}\left(r_{2}, r_{2}\right) \\
& +\frac{\omega_{1}+\lambda_{1}}{\Delta}\left\|\bar{\phi}_{1}\right\|_{1} \Phi_{1}\left(r_{2}, r_{2}\right) \\
& +t^{2-\alpha} \int_{0}^{t} \frac{(t-s)^{\alpha-1}}{\Gamma(\alpha)} s^{k_{1}}(1-s)^{l_{1}} \mathrm{~d} s \Phi\left(r_{2}, r_{2}\right) \\
& +\frac{v_{1}+\mu_{1}}{\Delta} \int_{0}^{1} \frac{(1-s)^{\alpha-1}}{\Gamma(\alpha)} s^{k_{1}}(1-s)^{l_{1}} \mathrm{~d} s \Phi\left(r_{2}, r_{2}\right) \\
& +\frac{b v_{1}+b \mu_{1}}{\Delta} \int_{0}^{\eta} \frac{(\eta-s)^{\alpha-1}}{\Gamma(\alpha)} s^{k_{1}}(1-s)^{l_{1}} \mathrm{~d} s \Phi\left(r_{2}, r_{2}\right) \\
& +\frac{a \lambda_{1}+a \omega_{1}}{\Delta} \int_{0}^{\xi} \frac{(\xi-s)^{\alpha-1}}{\Gamma(\alpha)} s^{k_{1}}(1-s)^{l_{1}} \mathrm{~d} s \Phi\left(r_{2}, r_{2}\right) \\
\leq & \frac{v_{1}+\mu_{1}}{\Delta}\left\|\bar{\psi}_{1}\right\|_{1} \Psi_{1}\left(r_{2}, r_{2}\right) \\
& +\frac{\omega_{1}+\lambda_{1}}{\Delta}\left\|\bar{\phi}_{1}\right\|_{1} \Phi_{1}\left(r_{2}, r_{2}\right) \\
& +\left[1+\frac{v_{1}+\mu_{1}}{\Delta}+\frac{b v_{1}+b \mu_{1}}{\Delta} \eta^{\alpha+k_{1}+l_{1}}\right. \\
& \left.+\frac{a \lambda_{1}+a \omega_{1}}{\Delta} \xi^{\alpha+k_{1}+l_{1}}\right] \frac{\mathbf{B}\left(\alpha+l_{1}, k_{1}+1\right)}{\Gamma(\alpha)} \Phi\left(r_{2}, r_{2}\right)
\end{aligned}
$$

and similarly we get

$$
\begin{aligned}
& t^{2+m-\alpha}\left|D_{0^{+}}^{m}\left(T_{1} y\right)(t)\right| \\
& \leq \quad \frac{v_{1} \frac{\Gamma(\alpha)}{\Gamma(\alpha-m)}+\mu_{1} \frac{\Gamma(\alpha-1)}{\Gamma(\alpha-m-1)}}{\Delta}\left\|\bar{\psi}_{1}\right\|_{1} \Psi_{1}\left(r_{2}, r_{2}\right) \\
& \quad+\frac{\omega_{1} \frac{\Gamma(\alpha-1)}{\Gamma(\alpha-m-1)}+\lambda_{1} \frac{\Gamma(\alpha)}{\Gamma(\alpha-m)}}{\Delta}\left\|\bar{\phi}_{1}\right\|_{1} \Phi_{1}\left(r_{2}, r_{2}\right) \\
& \quad+t^{2+m-\alpha} \int_{0}^{t} \frac{(t-s)^{\alpha-m-1}}{\Gamma(\alpha-m)} s^{k_{1}}(1-s)^{l_{1}} \mathrm{~d} s \Phi\left(r_{2}, r_{2}\right) \\
& \quad+\frac{v_{1} \frac{\Gamma(\alpha)}{\Gamma(\alpha-m)}+\mu_{1} \frac{\Gamma(\alpha-1)}{\Gamma \Gamma(\alpha-m-1) \mid}}{\Delta} \\
& \quad \times \int_{0}^{1} \frac{(1-s)^{\alpha-1}}{\Gamma(\alpha)} s^{k_{1}}(1-s)^{l_{1}} \mathrm{~d} s \Phi\left(r_{2}, r_{2}\right) \\
& \quad+\frac{b v_{1} \frac{\Gamma(\alpha)}{\Gamma(\alpha-m)}+b \mu_{1} \frac{\Gamma(\alpha-1)}{\Gamma(\alpha-m-1) \mid}}{\Delta} \\
& \quad \times \int_{0}^{\eta} \frac{(\eta-s)^{\alpha-1}}{\Gamma(\alpha)} s^{k_{1}}(1-s)^{l_{1}} \mathrm{~d} s \Phi\left(r_{2}, r_{2}\right) \\
& \quad+\frac{a \lambda_{1} \frac{\Gamma(\alpha)}{\Gamma(\alpha-m)}+a \omega_{1} \frac{\Gamma(\alpha-1)}{\Gamma(\alpha-m-1) \mid}}{\Delta}
\end{aligned}
$$




$$
\begin{aligned}
& \times \int_{0}^{\xi} \frac{(\xi-s)^{\alpha-1}}{\Gamma(\alpha)} s^{k_{1}}(1-s)^{l_{1}} \mathrm{~d} s \Phi\left(r_{2}, r_{2}\right) \\
& \leq \frac{v_{1} \frac{\Gamma(\alpha)}{\Gamma(\alpha-m)}+\mu_{1} \frac{\Gamma(\alpha-1)}{\Gamma(\alpha-m-1)}}{\Delta}\left\|\bar{\psi}_{1}\right\|_{1} \Psi_{1}\left(r_{2}, r_{2}\right) \\
& +\frac{\omega_{1} \frac{\Gamma(\alpha-1)}{\Gamma(\alpha-m-1)}+\lambda_{1} \frac{\Gamma(\alpha)}{\Gamma(\alpha-m)}}{\Delta}\left\|\bar{\phi}_{1}\right\|_{1} \Phi_{1}\left(r_{2}, r_{2}\right) \\
& +\left[\frac{\mathbf{B}\left(\alpha-m+l_{1}, k_{1}+1\right)}{\Gamma(\alpha-m)}+\frac{v_{1} \frac{\Gamma(\alpha)}{\Gamma(\alpha-m)}+\mu_{1} \frac{\Gamma(\alpha-1)}{|\Gamma(\alpha-m-1)|}}{\Delta}\right. \\
& \times \frac{\mathbf{B}\left(\alpha+l_{1}, k_{1}+1\right)}{\Gamma(\alpha)}
\end{aligned}
$$

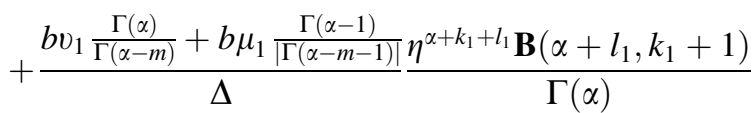

$$
\begin{aligned}
& +\frac{a \lambda_{1} \frac{\Gamma(\alpha)}{\Gamma(\alpha-m)}+a \omega_{1} \frac{\Gamma(\alpha-1)}{|\Gamma(\alpha-m-1)|}}{\Delta} \\
& \left.\frac{\xi^{\alpha+k_{1}+l_{1}} \mathbf{B}\left(\alpha+l_{1}, k_{1}+1\right)}{\Gamma(\alpha)} \Gamma(\alpha)\right] \Phi\left(r_{2}, r_{2}\right) .
\end{aligned}
$$

We get

$$
\begin{aligned}
& \left\|T_{1} y\right\| \leq \frac{v_{1}+\mu_{1}}{\Delta}\left\|\bar{\psi}_{1}\right\|_{1} \Psi_{1}\left(r_{2}, r_{2}\right)+\frac{\omega_{1}+\lambda_{1}}{\Delta}\left\|\bar{\phi}_{1}\right\|_{1} \Phi_{1}\left(r_{2}, r_{2}\right) \\
& +\left[1+\frac{v_{1}+\mu_{1}}{\Delta}+\frac{b v_{1}+b \mu_{1}}{\Delta} \eta^{\alpha+k_{1}+l_{1}}\right. \\
& \left.+\frac{a \lambda_{1}+a \omega_{1}}{\Delta} \xi^{\alpha+k_{1}+l_{1}}\right] \frac{\mathbf{B}\left(\alpha+l_{1}, k_{1}+1\right)}{\Gamma(\alpha)} \Phi\left(r_{2}, r_{2}\right) \\
& +\frac{v_{1} \frac{\Gamma(\alpha)}{\Gamma(\alpha-m)}+\mu_{1} \frac{\Gamma(\alpha-1)}{\Gamma(\alpha-m-1)}}{\Delta}\left\|\bar{\psi}_{1}\right\|_{1} \Psi_{1}\left(r_{2}, r_{2}\right) \\
& +\frac{\omega_{1} \frac{\Gamma(\alpha-1)}{\Gamma(\alpha-m-1)}+\lambda_{1} \frac{\Gamma(\alpha)}{\Gamma(\alpha-m)}}{\Delta}\left\|\bar{\phi}_{1}\right\|_{1} \Phi_{1}\left(r_{2}, r_{2}\right) \\
& +\left[\frac{\mathbf{B}\left(\alpha-m+l_{1}, k_{1}+1\right)}{\Gamma(\alpha-m)}\right. \\
& +\frac{v_{1} \frac{\Gamma(\alpha)}{\Gamma(\alpha-m)}+\mu_{1} \frac{\Gamma(\alpha-1)}{\Gamma(\alpha-m-1) \mid}}{\Delta} \frac{\mathbf{B}\left(\alpha+l_{1}, k_{1}+1\right)}{\Gamma(\alpha)}
\end{aligned}
$$

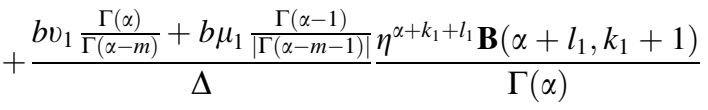

$$
\begin{aligned}
& +\frac{a \lambda_{1} \frac{\Gamma(\alpha)}{\Gamma(\alpha-m)}+a \omega_{1} \frac{\Gamma(\alpha-1)}{|\Gamma(\alpha-m-1)|}}{\Delta} \\
& \left.\times \frac{\xi^{\alpha+k_{1}+l_{1}} \mathbf{B}\left(\alpha+l_{1}, k_{1}+1\right)}{\Gamma(\alpha)} \Gamma(\alpha)\right] \Phi\left(r_{2}, r_{2}\right) \\
& =M_{1} \Psi_{1}\left(r_{2}, r_{2}\right)+N_{1} \Phi_{1}\left(r_{2}, r_{2}\right)+Q_{1} \Phi\left(r_{2}, r_{2}\right) \text {. }
\end{aligned}
$$

Similarly, we get

$$
\left\|T_{2} x\right\| \leq M_{2} \Psi_{2}\left(r_{1}, r_{1}\right)+N_{2} \Phi_{1}\left(r_{1}, r_{1}\right)+Q_{2} \Psi\left(r_{1}, r_{1}\right) .
$$

Since (13) has positive solution $r_{1}>0, r_{2}>0$, we choose $\Omega=\left\{(x, y) \in E:\|x\| \leq r_{1},\|y\| \leq r_{2}\right\}$. Then we get $T(\Omega) \subset \Omega$. Hence, the Schauder's fixed point theorem implies that $T$ has a fixed point $(x, y) \in \Omega$. So $(x, y)$ is a positive solution of BVP(1).

The proof of Theorem 3.1 is completed.

Theorem 3.2 Suppose

(B2) there exists $\bar{\phi}_{i}, \bar{\psi}_{i} \in L^{1}(0,1)(i=1,2)$ and nonnegative constants $M_{\Phi}, M_{\Psi}, M_{\Phi_{i}}, M_{\Psi_{i}}(i=1,2)$ such that

$\left|f\left(t, \frac{u}{t^{2-\beta}}, \frac{v}{t^{2+n-\beta}}\right)\right| \leq M_{\Phi}, t \in(0,1), u, v \in \mathbb{R}$,

$\left|g\left(t, \frac{u}{t^{2-\alpha}}, \frac{v}{t^{2+m-\alpha}}\right)\right| \leq M_{\Psi}, t \in(0,1), u, v \in \mathbb{R}$,

$\left|\phi_{1}\left(t, \frac{u}{t^{2-\beta}}, \frac{v}{t^{2+n-\beta}}\right)\right| \leq \bar{\phi}_{1}(t) M_{\Phi_{1}}, t \in(0,1), u, v \in \mathbb{R}$,

$\left|\psi_{1}\left(t, \frac{u}{t^{2-\beta}}, \frac{v}{t^{2+n-\beta}}\right)\right| \leq \bar{\psi}_{1}(t) M_{\Psi_{1}}, t \in(0,1), u, v \in \mathbb{R}$,

$\left|\phi_{2}\left(t, \frac{u}{t^{2-\alpha}}, \frac{v}{t^{2+m-\alpha}}\right)\right| \leq \bar{\phi}_{2}(t) M_{\Phi_{2}}, t \in(0,1), u, v \in \mathbb{R}$,

$\left|\psi_{2}\left(t, \frac{u}{t^{2-\alpha}}, \frac{v}{t^{2+m-\alpha}}\right)\right| \leq \bar{\psi}_{2}(t) M_{\Psi_{2}}, t \in(0,1), u, v \in \mathbb{R}$.

Then BVP(1) has at least one positive solution.

Proof Let $M_{i}, N_{i}, Q_{i}(i=1,2)$ be defined in Theorem 3.1. Choose $\Phi(u, v)=M_{\Phi}, \Psi(u, v)=M_{\Psi}, \Phi_{i}(u, v)=M_{\Phi_{i}}$ and $\Psi_{i}(u, v)=M_{\Psi_{i}}(i=1,2)$. We see that (13) has positive solution

$$
\begin{aligned}
& r_{1}=M_{1} M_{\Psi_{1}}+N_{1} M_{\Phi_{1}}+Q_{1} M_{\Phi}, \\
& r_{2}=M_{2} M_{\Psi_{2}}+N_{2} M_{\Phi_{1}}+Q_{2} M_{\Psi} .
\end{aligned}
$$

The results follows from Theorem 3.1 directly.

\section{Numerical examples}

In this section, we present two examples for the illustration of our main result (Theorems 3.1 and 3.2).

Example 4.1 We consider the following boundary value problem

$$
\left\{\begin{array}{l}
D_{0^{+}}^{\frac{19}{10}} u(t)+t^{-\frac{1}{10}}(1-t)^{-\frac{17}{20}} f\left(t, v(t), D_{0^{+}}^{\frac{39}{40}} v(t)\right)=0, \quad t \in(0,1) \\
D_{0^{+}}^{\frac{39}{20}} v(t)+t^{-\frac{1}{10}}(1-t)^{-\frac{13}{20}} g\left(t, u(t), D_{0^{+}}^{\frac{19}{20}} u(t)\right)=0, \quad t \in(0,1) \\
\lim _{t \rightarrow 0} t^{\frac{1}{5}} u(t)-\frac{1}{2} u(1 / 2)=0 \\
u(1)-\frac{1}{2} u(3 / 4)=0 \\
\lim _{t \rightarrow 0} t^{\frac{1}{9}} v(t)-\frac{1}{2} v(1 / 2)=0 \\
v(1)-\frac{1}{2} v(3 / 4)=0
\end{array}\right.
$$

Then

(i) $\operatorname{BVP}(13)$ has at least one positive solution if there exists a constant $H>0$ such that 


$$
\begin{aligned}
& \left|f\left(t, t^{-\frac{1}{20}} u, t^{-\frac{41}{40}} v\right)\right| \leq H, \quad t \in(0,1), u, v \in \mathbb{R}, \\
& \left|g\left(t, t^{-\frac{1}{10}} u, t^{-\frac{21}{20}} v\right)\right| \leq H, \quad t \in(0,1), u, v \in \mathbb{R} .
\end{aligned}
$$

$\mathrm{BVP}(13)$ has at least one positive solution if

$$
\begin{aligned}
& \left|f\left(t, t^{-\frac{1}{20}} u, t^{-\frac{41}{40}} v\right)\right| \leq H, t \in(0,1), u, v \in \mathbb{R}, \\
& \begin{aligned}
\left|g\left(t, t^{-\frac{1}{10}} u, t^{-\frac{21}{20}} v\right)\right| & \leq H, t \in(0,1), u, v \in \mathbb{R}, \\
\phi_{1}\left(t, t^{-\frac{1}{20}} u, t^{-\frac{41}{40}} v\right) & =\psi_{1}\left(t, t^{-\frac{1}{20}} u, t^{-\frac{41}{40}} v\right) \\
& =\phi_{2}\left(t, t^{-\frac{1}{10}} u, t^{-\frac{12}{20}} v\right) \\
& =\psi_{2}\left(t, t^{-\frac{1}{10}} u, t^{-\frac{21}{20}} v\right)=0,
\end{aligned}
\end{aligned}
$$

$\left|f\left(t, t^{-\frac{1}{20}} u, t^{-\frac{41}{40}} v\right)\right| \leq c_{1}+b_{1}|u|^{\epsilon_{1}}+a_{1}|v|^{\delta_{1}}, c_{1}, b_{1}, a_{1} \geq 0, \epsilon_{1}, \delta_{1}>0$,

$\left|g\left(t, t^{-\frac{1}{10}} u, t^{-\frac{21}{20}} v\right)\right| \leq c_{2}+b_{2}|u|^{\sigma_{1}}+a_{2}|v|^{\gamma_{1}}, c_{2}, b_{2}, a_{2} \geq 0, \sigma_{1}, \gamma_{1}>0$

and one of the followings holds:

(a) $\max \left\{\epsilon_{1}, \delta_{1}\right\} \max \left\{\sigma_{1}, \gamma_{1}\right\}<1$;

(b) $\max \left\{\epsilon_{1}, \delta_{1}\right\} \max \left\{\sigma_{1}, \gamma_{1}\right\}=1$ with $\left(38.1089 b_{1}\right)^{1 / \sigma_{1}}$ $34.0678 b_{2}<1$ or $38.1089 b_{1}\left(34.0678 b_{2}\right)^{1 / \tau_{1}}<1$

(c) $\max \left\{\epsilon_{1}, \delta_{1}\right\} \max \left\{\sigma_{1}, \gamma_{1}\right\}>1$ for sufficiently small $b_{1}, a_{1}, b_{2}, a_{2}$.

Proof Corresponding to BVP(1), we have $\alpha=\frac{19}{10}, \beta=\frac{39}{20}$, $m=\frac{19}{20}$ and $n=\frac{39}{40}, \xi=\frac{1}{2}, \eta=\frac{3}{4}, a=b=c=d=\frac{1}{2}$ and $\phi_{i}(t, u, v)=\psi_{i}(t, u, v) \equiv 0(i=1,2)$ and $p(t)=t^{-\frac{1}{10}}(1-$ $t)^{-\frac{17}{20}}, q(t)=t^{-\frac{1}{10}}(1-t)^{-\frac{13}{20}}$.

It is easy to see that (i)-(iv) hold with $k_{1}=-\frac{1}{10}=k_{2}$, and $l_{1}=-\frac{17}{20}, l_{2}=-\frac{13}{20}$. One sees that $k_{1}>-1$, $\alpha-m+l_{1}>0, \quad 2+k_{1}+l_{1}>0, \quad k_{2}>-1, \quad \beta-n+l_{2}$ $>0,2+k_{2}+l_{2}>0$. Hence, (i)-(iv) defined in Sect. 1 hold.

By direct calculation using Matlab7, we find that

$$
\begin{aligned}
& \mu_{1}=\frac{1}{2}\left(\frac{1}{2}\right)^{\frac{9}{10}}, \quad v_{1}=1-\frac{1}{2}\left(\frac{1}{2}\right)^{-\frac{1}{10}}, \\
& \omega_{1}=1-\frac{1}{2}\left(\frac{3}{4}\right)^{\frac{9}{10}}, \quad \lambda_{1}=1-\frac{1}{2}\left(\frac{3}{4}\right)^{-\frac{1}{10}}, \\
& \mu_{2}=\frac{1}{2}\left(\frac{1}{2}\right)^{\frac{19}{20}}, \quad v_{2}=1-\frac{1}{2}\left(\frac{1}{2}\right)^{-\frac{1}{20}}, \\
& \omega_{2}=1-\frac{1}{2}\left(\frac{3}{4}\right)^{\frac{19}{20}}, \quad \lambda_{2}=1-\frac{1}{2}\left(\frac{3}{4}\right)^{-\frac{1}{20}}
\end{aligned}
$$

and

$$
\begin{array}{ll}
\Delta=\mu_{1} \lambda_{1}+v_{1} \omega_{1}>0, \quad 0 \leq a<\frac{1}{\xi^{\alpha-2}(1-\xi)}, & 0 \leq b<\frac{1}{\eta^{\alpha-1}} \\
\nabla=\mu_{2} \lambda_{2}+v_{2} \omega_{2}>0, & 0 \leq c<\frac{1}{\xi^{\beta-2}(1-\xi)}, \quad 0 \leq d<\frac{1}{\eta^{\beta-1}}
\end{array}
$$

(i) $\mathrm{By}$
It follows from Theorem 3.2 that BVP(13) has at least one positive solution.

(ii) One sees that (B1) holds with

$$
\begin{aligned}
\Phi(u, v) & =c_{1}+b_{1}|u|^{\epsilon_{1}}+a_{1} v^{\delta_{1}}, \\
\Psi(u, v) & =c_{2}+b_{2} u^{\sigma_{1}}+a_{2} v^{\gamma_{1}}, \\
\Phi_{i}(u, v) & =\Psi_{i}(u, v)=0(i=1,2)
\end{aligned}
$$

. Furthermore, we have by direct computation (use Mathlab7.0) that

$$
\begin{aligned}
& Q_{1}=\left[1+\frac{v_{1}+\mu_{1}}{\Delta}+\frac{b v_{1}+b \mu_{1}}{\Delta} \eta^{\alpha+k_{1}+l_{1}}\right. \\
& \left.+\frac{a \lambda_{1}+a \omega_{1}}{\Delta} \xi^{\alpha+k_{1}+l_{1}}\right] \frac{\mathbf{B}\left(\alpha+l_{1}, k_{1}+1\right)}{\Gamma(\alpha)} \\
& +\frac{\mathbf{B}\left(\alpha-m+l_{1}, k_{1}+1\right)}{\Gamma(\alpha-m)} \\
& +\frac{v_{1} \frac{\Gamma(\alpha)}{\Gamma(\alpha-m)}+\mu_{1} \frac{\Gamma(\alpha-1)}{|\Gamma(\alpha-m-1)|}}{\Delta} \\
& \times \frac{\mathbf{B}\left(\alpha+l_{1}, k_{1}+1\right)}{\Gamma(\alpha)} \\
& +\frac{b v_{1} \frac{\Gamma(\alpha)}{\Gamma(\alpha-m)}+b \mu_{1} \frac{\Gamma(\alpha-1)}{|\Gamma(\alpha-m-1)|}}{\Delta} \\
& \times \frac{\eta^{\alpha+k_{1}+l_{1}} \mathbf{B}\left(\alpha+l_{1}, k_{1}+1\right)}{\Gamma(\alpha)} \\
& +\frac{a \lambda_{1} \frac{\Gamma(\alpha)}{\Gamma(\alpha-m)}+a \omega_{1} \frac{\Gamma(\alpha-1)}{|\Gamma(\alpha-m-1)|}}{\Delta} \\
& \times \frac{\xi^{\alpha+k_{1}+l_{1}} \mathbf{B}\left(\alpha+l_{1}, k_{1}+1\right)}{\Gamma(\alpha)} \\
& \simeq 67.8769 \leq 68
\end{aligned}
$$

and 


$$
\begin{aligned}
Q_{2}= & {\left[1+\frac{v_{2}+\mu_{2}}{\nabla}+\frac{c v_{2}+d \mu_{2}}{\nabla} \eta^{\beta+k_{2}+l_{2}}\right.} \\
& +\frac{c \lambda_{2}+c \omega_{2}}{\nabla} \xi^{\left.\beta+k_{2}+l_{2}\right] \mathbf{B}\left(\beta+l_{2}, k_{2}+1\right)} \frac{\mathbf{P}(\beta)}{\Gamma\left(\beta-n+l_{2}, k_{2}+1\right)} \\
& +\frac{\mathbf{B}(\beta-n)}{\nabla} \\
& +\frac{v_{2} \frac{\Gamma(\beta)}{\Gamma(\beta-n)}+\mu_{2} \frac{\Gamma(\beta-1)}{\Gamma(\beta-n-1) \mid}}{\nabla} \\
& \times \frac{\mathbf{B}\left(\beta+l_{2}, k_{2}+1\right)}{\Gamma(\beta)} \\
& +\frac{d v_{2} \frac{\Gamma(\beta)}{\Gamma(\beta-n)}+d \mu_{2} \frac{\Gamma(\beta-1)}{\Gamma(\beta-n-1) \mid}}{\nabla} \\
& \times \frac{\eta^{\beta+k_{2}+l_{2} \mathbf{B}\left(\beta+l_{2}, k_{2}+1\right)}}{\Gamma(\beta)} \\
& +\frac{c \lambda_{2} \frac{\Gamma(\beta)}{\Gamma(\beta-n)}+c \omega_{2} \frac{\Gamma(\beta-1)}{\Gamma(\beta-n-1) \mid}}{\nabla} \\
& \times \frac{\xi^{\beta+k_{2}+l_{2}} \mathbf{B}\left(\beta+l_{2}, k_{2}+1\right)}{\Gamma(\beta)} \\
& \simeq 56.4653 \leq 57 .
\end{aligned}
$$

One sees that inequality system (13) has positive solutions if

$$
\begin{aligned}
& 68\left[c_{1}+b_{1} r_{2}^{\epsilon_{1}}+a_{1} r_{2}^{\delta_{1}}\right] \leq r_{1}, \\
& 57\left[c_{2}+b_{2} r_{1}^{\sigma_{1}}+a_{2} r_{1}^{\gamma_{1}}\right] \leq r_{2}
\end{aligned}
$$

has positive solutions. One sees that if

$$
\begin{aligned}
& 68\left[c_{1}+\left(b_{1}+a_{1}\right) r_{2}^{\max \left\{\epsilon_{1}, \delta_{1}\right\}}\right] \leq r_{1}, \\
& 57\left[c_{2}+\left(b_{2}+a_{2}\right) r_{1}^{\max \left\{\sigma_{1}, \gamma_{1}\right\}}\right] \leq r_{2}
\end{aligned}
$$

has positive solution $\left(r_{1}, r_{2}\right)$ with $r_{1}>1$, $r_{2}>1$, then (14) has positive solution $\left(\max \left\{1, r_{1}\right\}, \max \left\{1, r_{2}\right\}\right.$.

(ii)-(a) $\max \left\{\epsilon_{1}, \delta_{1}\right\} \max \left\{\sigma_{1}, \gamma_{1}\right\}<1$. It is easy to see that (14) has positive a positive solution $\left(r_{1}, r_{2}\right)$ with $r_{1}>0, r_{2}>0$. It follows from Theorem 3.1 that BVP(13) has at least one solution if one of the followings holds:

(ii)-(b) $\max \left\{\epsilon_{1}, \delta_{1}\right\} \max \left\{\sigma_{1}, \gamma_{1}\right\}=1$. One sees that $(14)^{\prime}$ becomes

$68\left[c_{1}+\left(b_{1}+a_{1}\right) r_{2}\right] \leq r_{1}, 57\left[c_{2}+\left(b_{2}+a_{2}\right) r_{1}\right] \leq r_{2}$.

It is easy to see that the latest inequality system holds for sufficiently large $r_{1}^{\prime}, r_{2}^{\prime}>0$ if $68 \times 57\left(a_{1}+b_{1}\right)\left(a_{2}+b_{2}\right)<1$. Hence (15) has positive solution $\left(\max \left\{1, r_{1}^{\prime}\right\}, \max \left\{1, r_{2}^{\prime}\right\}\right.$. Then BVP(1) has positive solution by Theorem 3.1.

$$
\begin{aligned}
& \max \left\{\epsilon_{1}, \delta_{1}\right\} \max \left\{\sigma_{1}, \gamma_{1}\right\}>1 \text {. By } \\
& \lim _{\left(a_{1}, b_{1}, c_{1}\right) \rightarrow(0,0,0)} Q_{1}\left[c_{1}+b_{1}|u|^{\epsilon_{1}}+a_{1} v^{\delta_{1}}\right] \\
& =\lim _{\left(a_{2}, b_{2}, c_{2}\right) \rightarrow(0,0,0)} Q_{2}\left[c_{2}+b_{2} u^{\sigma_{1}}+a_{2} v^{\gamma_{1}}\right]=0,
\end{aligned}
$$

we know that (15) has positive solution $\left(r_{1}, r_{2}\right)$ with $r_{i}>0$. Then Theorem 3.1 implies that $\mathrm{BVP}(1)$ has at least one positive solution if $a_{1}, b_{1}, c_{1}, a_{2}, b_{2}, c_{2}$ are sufficiently small. The proof is completed.

Example 4.2 We consider the following boundary value problem

$$
\left\{\begin{array}{l}
D_{0^{+}}^{\frac{19}{10}} u(t)+t^{-\frac{1}{2}}(1-t)^{-\frac{1}{5}} f\left(t, v(t), D_{0^{+}}^{\frac{39}{40}} v(t)\right)=0, \quad t \in(0,1), \\
D_{0^{+}}^{\frac{39}{20}} v(t)+t^{-\frac{1}{2}}(1-t)^{\frac{1}{10}} g\left(t, u(t), D_{0^{+}}^{\frac{10}{20}} u(t)\right)=0, \quad t \in(0,1), \\
\lim _{t \rightarrow 0} t^{\frac{1}{5}} u(t)-\frac{1}{2} u(1 / 2)=A, \quad u(1)-\frac{1}{2} u(3 / 4)=B, \\
\lim _{t \rightarrow 0} t^{\frac{1}{9}} v(t)-\frac{1}{2} v(1 / 2)=C, \quad v(1)-\frac{1}{2} v(3 / 4)=D,
\end{array}\right.
$$

where

$$
f(t, u, v)=t^{2}+\frac{b_{1} t^{\frac{1}{20}} u^{\epsilon_{1}}+a_{1} t^{\frac{41}{40}} v^{\delta_{1}}}{\sqrt{2} \sqrt{b_{1}^{2} t^{\frac{1}{10}} u^{2 \epsilon_{1}}+a_{1}^{2} t^{\frac{41}{20}} v^{2 \delta_{1}}+1}},{ }_{1},
$$$$
b_{1} \geq 0, \epsilon_{1}, \delta_{1}>0 \text {, }
$$

$$
g(t, u, v)=4 t^{5}+\frac{b_{2} t^{\frac{1}{10}} u^{\sigma_{1}}+a_{2} t^{\frac{21}{200}} v^{\gamma_{1}}}{\sqrt{2} \sqrt{b_{2}^{2} \frac{1}{t^{5}} u^{2 \sigma_{1}}+a_{2}^{2} t^{\frac{21}{10}} v^{2 \gamma_{1}}+1}}, a_{2},
$$$$
b_{2} \geq 0, \sigma_{1}, \gamma_{1}>0 \text {. }
$$

Then BVP(15) has at least one positive solution for sufficiently small $a_{i}, b_{i}(i=1,2)$.

Proof Corresponding to BVP(1), we have $\alpha=\frac{19}{10}, \beta=\frac{39}{20}$, $m=\frac{19}{20}$ and $n=\frac{39}{40}, a=b=c=d=\frac{1}{2}$ and $\phi_{1}(t, u, v)=$ $A, \psi_{1}(t, u, v)=B, \phi_{2}(t, u, v)=C, \psi_{2}(t, u, v)=D$ and $p(t)$ $=t^{-\frac{1}{2}}(1-t)^{-\frac{1}{5}}, q(t)=t^{-\frac{1}{2}}(1-t)^{\frac{1}{10}}$.

It is easy to see that (i)-(iv) hold with $k_{1}=-\frac{1}{10}=k_{2}$, and $l_{1}=-\frac{1}{5}, \quad l_{2}=-\frac{1}{10}$. One sees that $k_{1}>-1$, $\alpha-m+l_{1}>0, \quad 2+k_{1}+l_{1}>0, \quad k_{2}>-1, \quad \beta-n+l_{2}$ $>0,2+k_{2}+l_{2}>0$. One sees $m>\alpha-1, n>\beta-1$.

Then similar to Example 4.1, we know that BVP(15) has at least one positive solution by Theorem 3.2. 


\section{Conclusions}

In this paper, we establish sufficient conditions for the existence of positive solutions of four-point integral type boundary value problems for singular fractional differential systems. We allow the nonlinearities $p(t) f(t, x, y)$ and $q(t) g(t, x, y)$ in fractional differential equations to be singular at $t=0$. Both $f$ and $g$ may be super-linear and sublinear. The analysis relies on some well known fixed point theorems. This paper contributes within the domain of fractional differential equations. The methods can be applied to solve other kinds of four-point integral type boundary value problems for singular fractional differential systems.

In $[12,22]$, authors studied the existence of positive solutions of two-point boundary value problems for fractional order elastic beam equations. One can discuss the following boundary value problem for nonlinear singular coupled fractional order elastic beam equations of the form

$$
\left\{\begin{array}{l}
D_{0^{+}}^{\alpha} u(t)=f\left(t, v(t), v^{\prime}(t), v^{\prime \prime}(t)\right), t \in(0,1), \\
D_{0^{+}}^{\beta} v(t)=g\left(t \cdot u(t), u^{\prime}(t), u^{\prime \prime}(t)\right), t \in(0,1), \\
\lim _{t \rightarrow 0} t^{4-\alpha} u(t)=\lim _{t \rightarrow 0} t^{4-\alpha} u^{\prime}(t)=0, \\
u(1)=u^{\prime}(1)=0, \\
\lim _{t \rightarrow 0} t^{4-\beta} v(t)=\lim _{t \rightarrow 0} t^{4-\beta} v^{\prime}(t)=0, \\
v(1)=v^{\prime}(1)=0,
\end{array}\right.
$$

where $3<\alpha, \beta \leq 4, D_{0^{+}}^{*}$ ( $D^{*}$ for short) is the RiemannLiouville fractional derivative of order $*$, and $f, g:(0,1) \times$ $[0, \infty) \times \mathbb{R}^{2} \rightarrow[0, \infty)$ is continuous. $f, g$ depend on the lower order fractional derivatives $u^{\prime}, v^{\prime}$ and $u^{\prime \prime}, v^{\prime \prime}$ and may be singular at $t=0$ and $t=1, f, g$ are non-Carathéodory functions.

Acknowledgements The author would like to thank the referees and the editors for their careful reading and some useful comments on improving the presentation of this paper.

Open Access This article is distributed under the terms of the Creative Commons Attribution 4.0 International License (http://crea tivecommons.org/licenses/by/4.0/), which permits unrestricted use, distribution, and reproduction in any medium, provided you give appropriate credit to the original author(s) and the source, provide a link to the Creative Commons license, and indicate if changes were made.

\section{References}

1. Ahmad, B., Nieto, J.J.: Existence results for a coupled system of nonlinear fractional differential equations with three-point boundary conditions. Comput. Math. Appl. 58, 1838-1843 (2009)

2. Avery, R.I., Peterson, A.C.: Three positive fixed points of nonlinear operators on ordered banach spaces. Comput. Math. Appl. 42, 313-322 (2001)
3. Basset, A.B.: On the descent of a sphere in a vicous liquid. Q. J. Pure Appl. Math. 41, 369-381 (1910)

4. Bai, C.Z., Fang, J.X.: The existence of a positive solution for a singular coupled system of nonlinear fractional differential equations. Appl. Math. Comput. 150(3), 611-621 (2004)

5. Caponetto, R., Dongona, G., Fortuna, L.: Fractional Differential Systems, Modeling and control applications, World Scientific Series on Nonlinear Science, Ser. A, vol. 72. World Scientific Publishing Co. Pte. Ltd. (2010)

6. Duan, J., Temuer, C.: Solution for system of linear fractional differential equations with constant coefficients. J. Math. 29, 599-603 (2009)

7. Gaber, M., Brikaa, M.G.: Existence results for a coupled system of nonlinear fractional differential equation with four-point boundary conditions. ISRN Math. Anal. Article ID 468346, pp 14 (2011)

8. Goodrich, C.S.: Existence of a positive solution to systems of differential equations of fractional order. Comput. Math. Appl. 62, 1251-1268 (2011)

9. Liu, Y.: Existence and non-existence of positive solutions of BVPs for fractional order elastic beam equations with a nonCaratheodory nonlinearity. Appl. Math. Model. 38(2), 620-640 (2014)

10. Liu, Y.: Existence of positive solutions of fractional order elastic beam equation with a non-Carathodory nonlinearity. Math. Methods Appl. Sci. 39(6), 1311-1324 (2015)

11. Liu, Y.: New existence results for positive solutions of boundary value problems for coupled systems of multi-term fractional differential equations. Hacet. Univ. Bull. Nat. Sci. Eng. 2(45), 391-416 (2016)

12. Liang, S., Zhang, J.: Positive solutions for boundary value problems of nonlinear frac- tional differential equations. Nonlinear Anal. 71, 5545-5550 (2009)

13. Liu, L., Zhang, X., Wu, Y.: On existence of positive solutions of a two-point boundary value problem for a nonlinear singular semipositone system. Appl. Math. Comput. 192, 223-232 (2007)

14. Mainardi, F.: Fraction Calculus: Some basic problems in continuum and statistical machanics. In: Carpinteri, A., Mainardi, F. (eds.) Fratals and Fractional Calculus in Continuum Machanics, pp. 291-348. Springer, Vien (1997)

15. Miller, K.S., Ross, B.: An Introduction to the Fractional Calculus and Fractional Differential Equation. Wiley, New York (1993)

16. Mamchuev, M.O.: Boundary value problem for a system of fractional partial differential equations. Partial Differ. Equ. 44, 1737-1749 (2008)

17. Rehman, M., Khan, R.: A note on boundaryvalueproblems for a coupled system of fractionaldifferential equations. Comput. Math. Appl. 61, 2630-2637 (2011)

18. Su, X.: Boundary value problem for a coupled system of nonlinear fractional differential equations. Appl. Math. Lett. 22(1), 64-69 (2009)

19. Torvik, P.J., Bagley, R.L.: On the appearance of the fractional derivative in the behavior of real materials. J. Appl. Mech. 51, 294-298 (1984)

20. Trujillo, J.J., Rivero, M., Bonilla, B.: On a Riemann-Liouville generalized Taylor's formula. J. Math. Anal. Appl. 231, 255-265 (1999)

21. Wang, J., Xiang, H., Liu, Z.: Positive solution to nonzero boundary values problem for a coupled system of nonlinear fractional differential equations. Int. J. Differ. Equ. Article ID 186928, pp 12. (2010). doi:10.1155/2010/186928

22. Xu, X., Jiang, D., Yuan, C.: Multiple positive solutions for the boundary value problem of a nonlinear fractional differential equation. Nonlinear Anal. 71, 4676-4688 (2009)

23. Yuan, C.: Multiple positive solutions for (n-1, 1)-type semipositone conjugate boundary value problems for coupled systems 
of nonlinear fractional differential equations, E. J. Qual. Theory of Differ. Equ. 13, 1-12 (2011)

24. Yang, A., Ge, W.: Positive solutions for boundary value problems of N-dimension nonlinear fractional differential systems, Boundary Value Problems, article ID 437453. (2008). doi:10. 1155/2008/437453

25. Yuan, C., Jiang, D., O'Regan, D., Agarwal, R.P.: Existence and uniqueness of positive solutions of boundary value problems for coupled systems of singular second-order three-point non-linear differential and difference equations. Appl. Anal. 87, 921-932 (2008)

26. Yuan, C., Jiang, D., O'regan, D., Agarwal, R.P.: Multiple positive solutions to systems of nonlinear semipositone fractional differential equations with coupled boundary conditions. Eur. J. Qual. Theory Diff. Equ. 13, 1-17 (2012) 